Copyright (C1996, American Institute of Aeronautics and Astronautics, Inc.

AIAA Meeting Papers on Disc, June 1996

A9636675, AIAA Paper 96-2221

\title{
Status of the development and implementation of optical spectroscopic techniques on the DLR high enthalpy shock tunnel HEG
}

\author{
W. H. Beck \\ DLR, Inst. fuer Stroemungsmechanik, Goettingen, Germany \\ M. Wollenhaupt \\ DLR, Inst. fuer Stroemungsmechanik, Goettingen, Germany \\ M. Rosenhauer \\ DLR, Inst. fuer Stroemungsmechanik, Goettingen, Germany \\ T. Mueller \\ DLR, Inst. fuer Stroemungsmechanik, Goettingen, Germany \\ J. Jourdan \\ DLR, Inst. fuer Stroemungsmechanik, Goettingen, Germany
}

\section{AIAA, Advanced Measurement and Ground Testing Technology Conference, 19th, New Orleans, LA, June 17-20, 1996}

\begin{abstract}
An overview of the development and implementation of spectroscopic diagnostic techniques for high enthalpy facilities (with examples from HEG) is given. Spectrally and spatially resolved results with NO LIF at $193 \mathrm{~nm}$ on HEG are given for the free stream and for various models (European HERMES, Japanese HOPE, aerobraking configuration, cylinder, and sphere). The problems of luminosity, fluorescence quenching, nonlinear effects, and corrections needed for single-shot measurements are discussed with reference to the HEG results and to extensive tests carried out on a specially designed heated cell capable of providing controlled NO conditions in a flow system. Other spectroscopic techniques such as emission spectroscopy, tunable diode laser absorption, and flash lamp absorption are referred to briefly. (Author)
\end{abstract}




\title{
STATUS OF THE DEVELOPMENT AND IMPLEMENTATION OF OPTICAL SPECTROSCOPIC TECHNIQUES ON THE DLR HIGH ENTHALPY SHOCK TUNNEL HEG
}

\author{
W. H. Beck*, M. Wollenhaupt, M. Rosenhauer,T. Müller and J. Jourdan \\ Institute for Fluid Mechanics \\ German Aerospace Research Establishment DLR \\ Bunsenstrasse 10 \\ 37073 Göttingen Germany
}

\begin{abstract}
An overview of the development and implementation of spectroscopic diagnostic techniques for high enthalpy facilities (with examples from HEG) is given. Spectrally and spatially resolved results with NO LIF at $193 \mathrm{~nm}$ on HEG are given for the free stream and for various models (European HERMES, Japanese HOPE, aerobraking configuration, cylinder, sphere). The problems of luminosity, fluorescence quenching, non-linear effects and corrections needed for single shot measurements are discussed with reference to the HEG results and to extensive tests carried out on a specially designed heated cell capable of providing controlled NO conditions in a flow system. Other spectroscopic techniques such as emission spectroscopy, tunable diode laser absorption and flash lamp absorption are referred to briefly.
\end{abstract}

\section{INTRODUCTION}

The nature of the gas flows in a high enthalpy facility such as the HEG dictates which optical techniques are suitable to provide non-intrusive measurements of both the free stream and model flow properties. It has been inferred from calculations that even the free stream flow in HEG is in chemical (and perhaps vibrational) non-equilibrium - this can be clearly seen in Fig. 1 , where the values for various gas properties for equilibrium and non-equilibrium are compared ${ }^{1}$. Here $\mathrm{O}_{2}$ and $\mathrm{O}$, and the temperature and pressure differ for these two cases quite markedly. Hence it would seem logical, at least for a characterisation of the free stream flow, to concentrate one's efforts in the implementation of optical techniques to those which can measure these properties. Since concentrations of $\mathrm{O}_{2}$ and

\footnotetext{
${ }^{*}$ Member AIAA

Copyright 191996 by DLR

Published by the American Institute of Aeronautics and Astronautics, Inc. with permission.
}

$O$, and gas (and not surface) temperatures are required, it is further necessary to use techniques that are species specific and provide gas temperatures (rotational $\cong$ translational) directly. In short, spectroscopic methods are called for. (Similar arguments also apply to the choice of methods to probe flows around models.)

Several spectroscopic techniques have been used (or are being readied for use) on HEG. (Work on other non-spectroscopic techniques such as holographic interferometry and Schlieren will not be discussed here since it has been published elsewhere ${ }^{2}$.) Most efforts have been put into the implementation of laser induced fluorescence (LIF) of the species NO, which is found "naturally" in HEG free stream and model flows. The development of this work, up to the present status, will form the substance of this paper. The other spectroscopic techniques will be mentioned only briefly here.

\section{EXPERIMENTAL SETUP}

The HEG facility has been described in detail elsewhere ${ }^{3}$, so that only the run conditions in the nozzle reservoir and in the free stream are summarised here - see Table I. Almost all LIF tests on HEG were performed at Condition I (high enthalpy $h_{0}=21 \mathrm{MJ} \mathrm{kg}$, low pressure $p_{0}=39$ MPa).

The LIF apparatus shown in Fig. 2 has been described elsewhere ${ }^{4.5}$, so that only a brief description will be given here. Two counterpropagating excimer laser beams (ArF at around $193 \mathrm{~nm}$ ), tuned to two different transitions of NO, excite fluorescence from NO in different regions of the HEG flow (in the free-stream, behind shocks, in wakes). The fluorescence arising from each beam is detected separately by a gated, image-intensified CCD camera, either as a 2D image (PLIF) with the aid of special band pass filters, or as a spectrally resolved LIF image by placing a spectrometer between object plane and camera (optical multichannel analyser). Small amounts $(5 \%)$ are decoupled from each laser beam and passed through a heated calibration cell to fine tune the lasers before a test and to check on de-tuning during the test. 


\begin{tabular}{|c||cccc|}
\hline Condition & I & II & III & IV \\
\hline $\mathrm{p}_{\mathrm{o}}(\mathrm{MPa})$ & 38.63 & 90.85 & 44.97 & 111.10 \\
$\mathrm{~T}_{0}(\mathrm{~K})$ & 9055 & 9727 & 7279 & 8113 \\
$\mathrm{~h}_{\mathrm{o}}(\mathrm{MJ} / \mathrm{kg})$ & 21.06 & 22.30 & 13.19 & 14.84 \\
$\mathrm{p}(\mathrm{kPa})$ & 0.43 & 1.21 & 0.47 & 1.32 \\
$\mathrm{~T}(\mathrm{~K})$ & 790 & 1036 & 553 & 722 \\
$\rho\left(\mathrm{kg} / \mathrm{m}^{3}\right)$ & $1.64 \mathrm{e}-3$ & $3.59 \mathrm{e}-3$ & $2.83 \mathrm{e}-3$ & $6.15 \mathrm{e}-3$ \\
$\mathrm{M}$ & 9.70 & 9.03 & 9.98 & 9.48 \\
$\mathrm{u}(\mathrm{m} / \mathrm{s})$ & 5919 & 6157 & 4813 & 5148 \\
{$\left[\mathrm{e}^{-}\right](\mathrm{mol} / \mathrm{g})$} & $1.08 \mathrm{e}-8$ & $7.83 \mathrm{e}-9$ & $2.40 \mathrm{e}-9$ & $1.82 \mathrm{e}-9$ \\
{$\left[\mathrm{~N}_{2}\right](\mathrm{mol} / \mathrm{g})$} & $2.64 \mathrm{e}-2$ & $2.62 \mathrm{e}-2$ & $2.58 \mathrm{e}-2$ & $2.58 \mathrm{e}-2$ \\
{$\left[\mathrm{O}_{2}\right](\mathrm{mol} / \mathrm{g})$} & $1.11 \mathrm{e}-3$ & $2.01 \mathrm{e}-3$ & $4.45 \mathrm{e}-3$ & $4.87 \mathrm{e}-3$ \\
{$[\mathrm{Ar}](\mathrm{mol} / \mathrm{g})$} & $3.45 \mathrm{e}-4$ & $3.45 \mathrm{e}-4$ & $3.45 \mathrm{e}-4$ & $3.45 \mathrm{e}-4$ \\
{$[\mathrm{~N}](\mathrm{mol} / \mathrm{g})$} & $6.12 \mathrm{e}-8$ & $1.48 \mathrm{e}-8$ & $2.15 \mathrm{e}-8$ & $1.01 \mathrm{e}-8$ \\
{$[\mathrm{O}](\mathrm{mol} / \mathrm{g})$} & $1.12 \mathrm{e}-2$ & $9.07 \mathrm{e}-3$ & $3.34 \mathrm{e}-3$ & $2.49 \mathrm{e}-3$ \\
{$[\mathrm{NO}](\mathrm{mol} / \mathrm{g})$} & $1.10 \mathrm{e}-3$ & $1.41 \mathrm{e}-3$ & $2.27 \mathrm{e}-3$ & $2.27 \mathrm{e}-3$ \\
{$[\mathrm{NO}](\mathrm{mol} / \mathrm{g})$} & $1.08 \mathrm{e}-8$ & $7.83 \mathrm{e}-9$ & $2.40 \mathrm{e}-9$ & $1.82 \mathrm{e}-9$ \\
Molecular Weight $(\mathrm{g} / \mathrm{mol})$ & 24.93 & 26.60 & 27.64 & 27.96 \\
Reynolds No. $\left(\mathrm{x} / 0^{5} / \mathrm{m}\right)$ & 2.70 & 5.21 & 4.77 & 9.32 \\
\hline
\end{tabular}

Table 1. Nozzle reservoir and freestream conditions, as determined by calibration data and computed by the programs ESTC and STUB.

\section{NO LIF ON HEG}

The combination of two fluorescence images recorded in HEG flows to provide quantitative absolute temperatures has proved extremely difficult in single shot laser measurements using the NO transitions accessible in the ArF laser tuning range. The short tunnel test times dictate the use of a single shot technique, and the intense luminosity in the vicinity of model bow shocks necessitates the use of an NO transition whose fluorescence occurs in a wavelength region (a spectral gap) where the luminosity is weakest. For this reason, NO $D(0) \rightarrow X\left(v^{\prime \prime}\right)$ transitions excited at $193 \mathrm{~nm}$ with fluorescence between 193 and about $300 \mathrm{~nm}$ were, chosen. The advantage of these transitions is that they have strong transition moments (compared to, for example, the X A or X B transitions), and that they originate from vibrationally excited $(v=1)$ NO of the ground electronic $X$ state, which reduces the influences of laser beam absorption in its path through the test gas(es). The disadvantages in their use are the lower populations in the excited vibrational levels (leading to weaker fluorescence signals) and the multitude of $D \leftarrow X, B \leftarrow X$ and $A \leftarrow X$ transitions accessible to the laser at $193 \mathrm{~nm}$, making the search for suitable $D \leftarrow X$ lines difficult. Furthermore, the observed intensities of the satellite lines relative to the main branches show a non-Hönl-London behaviour, which at present cannot be explained, but could be due to saturation or excited state energy level interactions. These topics will be further addressed in 4.

\subsection{HEG FREE STREAM}

LIF measurements using the NO $R_{22}(27.5)$ and $R_{21}(17.5)\left(+P_{11}(35.5)\right)$ lines of the $\varepsilon(0,1)$ branch were carried out in the HEG free stream for Conditions I to IV (see Table I). The captured spectrally resolved fluorescence images covered the range 193 - $280 \mathrm{~nm}$. Calibration measurements were carried out using the heated cell placed in the test section and filled with a $1 \%$ $\mathrm{NO} / \mathrm{N}_{2}$ mixture at $800 \mathrm{~K}$ and $1 \mathrm{mbar}$ pressure, corresponding approximately to HEG free stream conditions (for these two bulk properties). Fig. 3 gives a comparison of measured fluorescence spectra from one of the two cameras for five HEG shots, showing both the primary $D(0) \rightarrow$ $X\left(v^{\prime \prime}\right)$ and cascade $A(0) \rightarrow X\left(v^{\prime \prime}\right)$ components. After applying laser tuning and intensity corrections, and combining with the calibration measurements, NO rotational temperatures $T_{e x}$ were determined using only the $D(0) \rightarrow$ $X\left(v^{4}=2,3,4\right)$ lines $^{6}$. The results are shown in Table 2.

A comparison between $T_{\text {ax }}$ and the calculated $T_{\text {th }}$ values, also expressed as a percentage of $T_{\text {th }}$ $\Delta T(\%)$, shows considerable non-systematic discrepancies. The reasons for this behaviour are still under study, and will be further discussed in 4.3. 


\begin{tabular}{|c|c|c|c|c|c|c|}
\hline $\begin{array}{c}\text { Shot } \\
\text { No. }\end{array}$ & $\begin{array}{c}\text { Cond- } \\
\text { ition }\end{array}$ & $\begin{array}{c}P_{n} \\
(\mathrm{MPa})\end{array}$ & $\begin{array}{c}\mathrm{h}_{\mathrm{o}} \\
(\mathrm{MJ} / \mathrm{kg}\end{array}$ & $\begin{array}{c}T_{\text {ih }} \\
(\mathrm{K})\end{array}$ & $\begin{array}{c}\mathrm{T}_{\text {(rr }} \\
(\mathrm{K})\end{array}$ & $\begin{array}{c}\Delta \mathrm{T} \\
(\%)\end{array}$ \\
\hline 140 & III & 44.7 & 13.2 & 550 & 690 & -25 \\
141 & II & 84.6 & 22.7 & 1030 & 710 & 30 \\
142 & I & 38.9 & 21.0 & 790 & 730 & 8 \\
143 & III & 40.0 & 12.0 & 470 & 880 & $-86(!)$ \\
145 & II & 62.5 & 20.9 & 930 & 560 & 40 \\
146 & IV & 100.0 & 14.4 & 640 & 680 & -6 \\
\hline
\end{tabular}

Table 2. Measured NO rotational temperatures $T_{a x}$ in the HEG free stream $\left(p_{0}=\right.$ reservoir pressure, $h_{0}=$ enthalpy, $T_{\text {th }}=$ calculated temperature ${ }^{3}, \Delta T=\%$ difference $T_{t h}$ to $T_{* x}$ )

\subsection{SPACE GLIDER HERMES}

Measurements ${ }^{6}$ were carried out in HEG at Condition 1 on a HERMES model (the previously planned but at present scrapped European space glider) placed at $40^{\circ}$ angle of attack in the test section and using a similar LIF configuration to before (3.1), but with the monochromator slit placed across the laser beam to give spatial resolution along a line upstream of the model. The experimental setup and a typical spectrally resolved LIF image are shown in Fig. 4. Also shown in the figure are spectral profiles in the two regions behind the shock (2) and in the free stream (3) (note the relative scaling!). The intense luminosity behind the shock has been analysed over a large spectral range (180 - 850 $\mathrm{nm}$ ) and been shown to be due largely to iron (Fe) emission ${ }^{6.7}$. In order to reduce the source of this emission, a full-length $(17 \mathrm{~m})$ single-piece copper liner is being installed in the shock tube; copper has a higher thermal product ( $\mathrm{ck}^{*}$ value) than steel, giving better heat conduction and capacity properties, and therefore leading to less ablation of material.

The emission results show that, at present, this luminosity at wavelengths above about $230 \mathrm{~nm}$ completely swamps the weaker laser induced $\mathrm{NO}$ fluorescence signals, whereas below $230 \mathrm{~nm}$ the luminosity, though still present, is considerably reduced in intensity. Therefore, in order to be able to carry out 2D spatially resolved fluorescence measurements in the vicinity of strong shocks, it is necessary to restrict one's attention to the small spectral region of $193-230$ $\mathrm{nm}$, and to suppress the higher wavelengths with the use of high blockage filters. The results also show that, under HEG conditions, laser excitation at around $226 \mathrm{~nm}$, leading to fluorescence at higher wavelengths, is not feasible. Furthermore, the necessity to filter the fluorescence results then brings up the question of how the energy is distributed amongst the other upper NO electronic energy levels after excitation to the $D$

$\rho \equiv$ density; $c \equiv$ specific heat; $k \equiv$ thermal conductivity level, and whether, by picking out just one or two of the $D$ emission lines, one can still combine two fluorescence signals to extract meaningful temperatures. This topic is further discussed in 4.3.

\subsection{AEROBRAKE CONFIGURATION WAKE}

A blunted cone model with heavily instrumented cylindrical afterbody (the sting) was tested in HEG to examine the wake flow and to study the wake closure. The model sting was equipped with a slit just downstream of the model attachment point, enabling two ArF laser sheets to counter propagate through the test section from both top and bottom behind the model, with both cameras being focussed onto the same overlapping region - see Fig. 5 . In this case, both laser beam cross sections were reduced in size to $2 \mathrm{~mm} \times 1.5 \mathrm{~mm}$ using a Kepler telescope arrangement, and the monochromator slit was placed along the beam direction for two locations behind the model, as shown in Fig. 5. The abovementioned NO line pair (see 3.1) was used, and a calibration measurement with heated cell placed in the test section was carried out after the tests. Four shots were performed, all at HEG condition III. Spectrally resolved images were obtained for all shots, but the subsequent calibration measurements showed ${ }^{5}$ that saturation effects using the focussed beams could not be neglected, so that an absolute temperature could not be determined. It was nevertheless possible to determine a relative temperature by assuming a calculated" value of $T_{\max }=1500 \mathrm{~K}$ at the top of location 1 (Fig. 5). The result $T / T_{\max }$ versus distance along the slit is shown in Fig. 6 for the two slit locations. The temperature change along these locations is as expected $^{2}$, and the reproducibility from shot to shot is shown to be good. The spatial resolution along the slit is unfortunately not good enough here to distinguish any flow effects (e.g. the shear layer).

Based on an analysis ${ }^{0,7}$ of the emission results from the stagnation region of a cylinder model (see 3.2), 2D spatially resolved LIF measurements of the blunt body wake region were carried out using tunable bandpass filters ${ }^{5}$ which gave access to the spectral gap at around $210 \mathrm{~nm}$, suppressing the intense luminosity found at higher wavelengths (see Fig. 7). Here the two laser beams were formed to sheets with dimensions $1.5 \mathrm{~mm} \times 40 \mathrm{~mm}$, and both were counter propagated through the sting slot, using the experimental configuration described before. In spite of these filters, the luminosity arising from the shock-heated gases around the model were still strong enough to swamp the weaker NO LIF 
signals in the immediate wake region. Attention was then focussed on a region further downstream of the model (reduced luminosity effects) where wake closure, as evidenced by impingement of the shear layer on the sting, occurs. Since there was no slot in the sting here, both laser beams were positioned to just graze the edge of the sting. LIF signals were weak here, but nevertheless the location of the recompression shock could be determined, see Fig. 8. Using the relative temperature method just described, and assuming the calculated temperature of $1000 \mathrm{~K}$ upstream of the recompression shock, the change of temperature behind the shock could be determined. This is shown also in Fig.8.

\section{3,4 CYLINDRICAL MODEL BOW SHOCK}

No LIFsignals could be detected in the stagnation region of the HERMES model (see 3.2), even in the wavelength region $(<230 \mathrm{~nm})$ where the luminosity intensity was considerably reduced. This could be due either to the still too strong luminosity, or to the known poor spatial resolution along the slit of the monochromator, which is further exacerbated by the small shock stand-off distance ( $<2 \mathrm{~mm}$ ) for this model. To check this, and to further examine the possibility of obtaining an LIF signal from behind a strong shock, measurements were carried out on a larger model, a cylinder of diameter $90 \mathrm{~mm}$ placed in the HEG test section with axis transverse to the flow $^{12}$. All shots were performed at condition I, where the shock stand-off distance was expected to be about $12-13 \mathrm{~mm}$, hence to some extent offsetting the rather poor spatial resolution along the monochromator slits, which here were placed across the laser sheets in a configuration similar to the HERMES tests (3.2). To obtain maximum signals, the strongest accessible mixed branch transition of NO - $\varepsilon(0,1)\left(Q_{11}(32.5)+Q_{21}(26.5)+\right.$ $R_{11}(26.5)+R_{21}(21.5)$ ) - was used.

Fig. 9 shows the result. The bright luminosity at all wavelengths behind the shock can be seen in region 2, and the expected and well characterised NO fluorescence is present in region 4 of the free stream. However, in region 3 , which is still in the free stream, one sees still an overlap of (spectrally unresolved) emission over the free stream NO fluorescence signal. This is thought to be due to scattering of the powerful emission arising from region 2 off contaminants found in the free stream in region 3. This scattered light falls off with distance from the shock; the boundary between region 3 and 4 is not sharp, and just mirrors the detectivity of NO fluorescence relative to the intensity of the scattered light. It turns out that a cylindrical model in the position used here is not suitable for LIF measurements; the fluorescence arising from the laser sheet behind the shock has to pass through the extended shocked region along the cylinder (corresponding to half the cylinder length of 450 $\mathrm{mm}$ ) before reaching the cameras, leading to a greater luminosity component and, potentially, to self-absorption of the fluorescence by NO (especially for lower vibrational levels $v$ ) in this shocked region. (To help alleviate these problems, future measurements were carried out with a spherical model (see 3.6).)

\subsection{JAPANESE SPACE GLIDER HOPE}

Double and power delta models, two potential configurations for the planned Japanese space plane HOPE, were tested at angle of attack $35^{\circ}$ and yaw angles $\beta-3,0$ and $+3^{\circ}$ in HEG at conditions I, II and IV. For both models, the interaction of the bow shock with the winglet and/or the wing leading edge was of particular interest, so that flow visualisation using a sheet technique (as opposed to a line-of-sight integrating technique) such as PLIF was of great relevance. Hence, two LIF laser beams probed different but symmetrically disposed regions on opposite sides of the models, and the CCD cameras with bandpass filters $(200-220 \mathrm{~nm})$ captured the resulting $2 \mathrm{D}$ spatially resolved LIF images, as shown in Fig. 10.

Figs. 11a) and b) show LIF images at HEG condition I for the power and double delta models at $\beta=0$ and $+3^{\circ}$, respectively, overlapped with an image of the model itself, taken before the HEG shot using a flood lamp and long exposure times. The overlapping of LIF and calibration images and their scaling calibration, necessary to determine shock position and impingement point accurately, proved to be a most difficult task. Nevertheless, both images clearly show the position of the shock relative to the easily identifiable region of the model. In a procedure similar to that adopted for the aerobraking configuration (3.3), an attempt was also made to determine a temperature change across the shock relative to an assumed value for the free stream temperature $\mathrm{T}_{\mathrm{ts}}=760 \mathrm{~K}$, based on calculations using STUB ${ }^{13}$. The result, shown in Fig. 12, indicates a temperature jump from 760 to about $1500 \mathrm{~K}$, which is in good agreement with the value suggested by calculations based on the same $T_{t s}^{14}$. 


\subsection{SPHERICAL MODEL BOW SHOCK}

The failure so far to obtain an NO LIF signal behind the strong shocks with HERMES (3.2) and cylinder (3.4) models could be attributed to any one (or several) of the following:-

(i) luminosity of the hot gases at temperatures of around $9000 \mathrm{~K}$ is always so intense, even at $\lambda<230 \mathrm{~nm}$, that it swamps the weaker LIF signals;

(ii) the fluorescence is re-absorbed via resonant absorption by the hot NO present in the optical path (which can be very long, cf. cylinder) between object plane and detection system;

(iii) the laser beam and/or fluorescence is diverted by refraction after passing through the strong shock with its large density gradient ( $\rho / \rho_{-}>10$ for real gases);

(iv) quenching of fluorescence is very efficient due to the presence of potentially effective quenchers such as $\mathrm{O}$ atoms and hot $\mathrm{N}_{2}$, leading to vanishingly small quantum yields.

To (i):- As mentioned before, a copper liner will be installed in HEG to reduce the source of the spurious emission caused largely by $F e$ vapour. This probably won't remove the contaminating emission completely, but should help to reduce it considerably:

To (iv):- Experiments so far indicate that quenching (and other collision-induced energy transfer processes in NO) play a role in HEG, even in the free stream. Whether or not these processes influence a temperature measurement, where ratios of fluorescence signals arising from two different NO transitions are formed, still needs to be investigated (see 5). To (ii) and (iii):-in a comparable but not identical experimental setup (see Fig. 13(a)) to the cylinder measurements (3.4), spectrally resolved LIF signals were recorded in the stagnation region of a sphere of diameter $160 \mathrm{~mm}$ in HEG at condition I. The advantage of a sphere model is that the optical path for LIF signals through the shock-heated gases is much shorter than for the cylinder model, hence not only providing a check on (ii), but also reducing the luminosity component overlapping the LIF fluorescence signals. Furthermore, the laser beam was monitored by beam profile cameras (see Fig. 2) before and after passing through the test section, thereby giving an indication. of beam steering effects and beam absorption along its lengthy traversal path $(>0.5 \mathrm{~m}$ ) through the nozzle core flow (check of (iii)). Neither fluorescence reabsorption, beam steering nor beam absorption (by NO) were found to be a problem.
There were two further substantial differences in the setup here compared to the HERMES and cylinder work:- first, as opposed to the normal camera gates of $50-200 \mathrm{~ns}$, extremely short gates of only $5-10$ ns were used in an attempt to further suppress the intense luminosity. (This is most difficult due to the inherent jitter in laser firing and triggering electronics, leading to a risk of the laser pulse firing outside the gate of the cameras and losing the LIF signals completely.) Secondly, the laser beam energy density was decreased by de-focusing the beams in both directions, leading to dimensions of thickness 10 $\mathrm{mm}$ (cf. usually $1 \mathrm{~mm}$ ) and width $5 \mathrm{~mm}$ (usually $40 \mathrm{~mm}$ ), where thickness pertains to the direction along which the cameras integrate their signal; this is a check on non-linear (saturation) effects which can be brought about by too large energy densities. (The accompanying loss of spatial resolution was of little importance here.)

The experimental arrangement (a), a recorded LIF image (b) and spectral profiles (relative intensity versus wavelength) for the free stream and shocked regions (c) are shown in Fig. 13. Even though there is still strong luminosity in the image in Fig. 13(b) down to about $220 \mathrm{~nm}$, one can still discern an overlap of NO fluorescence lines which correlate quite well (especially below $220 \mathrm{~nm}$ ) with the lines seen in the free stream region of the same image. This is further reinforced by the spectral plot shown in Fig. $13(\mathrm{c})$. This result is the first case of a successful LIF measurement behind a strong shock in HEG; once the difficulties with single shot temperature determination using LIF (referred to before) have been overcome, the result shows that at least this most essential prerequisite for measurement of temperatures behind shocks in HEG is met. This task will be made even easier if the LIF signal intensity relative to the background luminosity is further improved by reducing the sources of contamination (and hence the intensity of background emission) in HEG.

\section{NO LIF ON THE CALIBRATION CELL}

It was recognised very early in the implementation of LIF on HEG that a constant source of hot NO would be necessary to study NO spectroscopy in the wavelength range of the ArF laser, to select and test suitable. NO transitions for determination of temperatures, to examine in detail the measurement procedure for temperature determination in HEG under more conducive conditions, and to study phenomena such as NO LIF quenching, saturation effects and energy transfer processes (via time resolved fluorescence detection). For this reason, a heated calibration cell was constructed ${ }^{4}$ to 
provide a continuous and homogeneous flow of NO at pressures $(1-10000 \mathrm{~Pa})$ and temperatures (at present up to $1300 \mathrm{~K}$ ) which could be easily adjusted. (These parameters are not always easy to adjust in a flame.)

\subsection{NO SPECTROSCOPY AT $193 \mathrm{NM}$}

For reasons already mentioned (see 3.), NO LIF work on HEG has concentrated on the wavelength range around $193 \mathrm{~nm}$. Although much work has been published ${ }^{15}$, there are still some uncertainties and discrepancies regarding the identification of all the lines in NO excitation spectra, especially at higher temperatures where myriads of "new" lines (arising from vibrationally excited levels of NO) appear. A complete analysis of NO spectra over the ArF laser tuning range was carried out, encompassing measurement of excitation and fluorescence (emission) spectra; the latter proved to be essential to enable an unequivocal identification of all seen transitions. In all, 400 transitions could be identified and tabulated ${ }^{4,16}$. Fig. 14 shows an NO excitation spectrum recorded at $1200 \mathrm{~K}$ and simulated spectra for the various branches of the $\varepsilon(0,1)$ and $\gamma(3,0 ; 4,1 ; 5,2)$ bands, and Fig. 15 shows the development in complexity in the excitation spectrum as the temperature is increased from 300 to $1214 \mathrm{~K}$.

\subsection{SELECTION OF SUITABLE LINE PAIRS}

Selection criteria for line pairs suitable for LIF need to be formulated ${ }^{5,16}$ :-

1. they must lie comfortably within the ArF laser locking range;

2. they must be well separated compared to the laser band width of $\sim 0.3 \mathrm{~cm}^{-1}$;

3. they should not lie in regions of strong (atmospheric) $\mathrm{O}_{2}$ absorption;

4. they should be strong lines in order to obtain sufficient fluorescence intensity - this is a -combined influence of transition moment and Boltzmann fraction;

5. the energy separation $\triangle E$ between their two source levels should be large relative to $k T$;

6. their fluorescence should occur at a wavelength not disturbed by other radiation.

(Criteria 1 . to 5 . apply generally to LIF measurements, 6. applies to HEG.) With these criteria as a basis, and making use of the spectral analysis referred to in 4.1 , tests were carried out in the hot cell at different temperatures (see Fig. 15) and pressures (20, 100 and $500 \mathrm{~Pa}$ ) to ascertain which line pairs are suitable. It was shown ${ }^{16}$ that the relative intensities of the $D \rightarrow X$ fluorescence lines exhibit an expected behaviour as pressure and temperature are varied, whereas the $A \rightarrow X$ cascade fluorescence lines are very temperature and pressure sensitive, indicating that the energy transfer processes leading to population of these A levels are also sensitive to these parameters. This can be crucial when spectral filtering is carried out. For this reason, in HEG, spectral filtering is restricted to the $D \rightarrow X$ lines only. In all, several candidate lines arising from $D(0)$, $A(3), A(4), B(7)$ and $A(5)$ levels, being of varying intensity, and in some cases not consisting of single transitions, were identified, and are shown in Table 3. Most work in HEG was carried out with the $R_{21}(17.5)$ and $R_{22}(27.5)$ line pair, for which $\Delta E$ is quite large $(\Delta E / K \sim 1310 K)$. (Even though there is an overlap of the $R_{2 s}(17.5)$ with a $P_{11}(35.5)$ line, this is not a problem for measurements at HEG free stream temperatures where the high $\mathrm{J}=35.5$ rotational level is hardly populated.)

\begin{tabular}{|c|c|c|c|}
\hline Band & Transition & Single line & $\begin{array}{l}\text { Relative } \\
\text { intensity }\end{array}$ \\
\hline$D(0)$ & $\begin{array}{l}Q_{m}(28.5) \\
P_{m}(36.5) \\
R_{m}(17.5) \\
Q_{m}(32.5) \\
R_{m}(27.5) \\
R_{m}(28.5) \\
R_{m}(29.5) \\
Q_{m}(32.5)\end{array}$ & $\begin{array}{c}\text { yes } \\
\text { (yes) } \\
\text { no } \\
\text { (yes) } \\
\text { yes } \\
\text { (yes) } \\
\text { (yes) } \\
\text { yes }\end{array}$ & $\begin{array}{c}3.7 \\
2.0 \\
12.5 \\
3.5 \\
10 \\
8.9 \\
5.2 \\
4.3\end{array}$ \\
\hline$A(3)$ & $\begin{array}{l}R_{n}(32.5) \\
Q_{11}(47.5) \\
R_{11}(40.5) \\
P_{11}(58.5) \\
R_{m b}(43.5) \\
R_{11}(43.5) \\
A_{m 0}(41.5) \\
R_{01}(41.5)\end{array}$ & $\begin{array}{l}\text { yes } \\
\text { yes } \\
\text { (yes) } \\
\text { yes } \\
\text { yes } \\
\text { (yes) } \\
\text { yes } \\
\text { yes }\end{array}$ & $\begin{array}{l}1.2 \\
1.9 \\
4.9 \\
0.3 \\
1.8 \\
5.5 \\
1.5 \\
1.3 \\
\end{array}$ \\
\hline$A(4)$ & $\begin{array}{l}R_{11}(15.5) \\
Q_{11}(23.5) \\
Q_{11}(26.5) \\
R_{11}(20.5) \\
Q_{11}(30.5) \\
R_{11}(17.5) \\
Q_{p 2}(33.5) \\
R_{11}(18.5) \\
R_{11}(24.5) \\
Q_{11}(33.5) \\
Q_{11}(29.5)\end{array}$ & $\begin{array}{l}\text { yes } \\
\text { yes } \\
\text { yes } \\
\text { yes } \\
\text { yes } \\
\text { yes } \\
\text { yes } \\
\text { (yes) } \\
\text { (yes) } \\
\text { yes } \\
\text { yes } \\
\end{array}$ & $\begin{array}{l}1.2 \\
2.2 \\
2.2 \\
5.0 \\
2.3 \\
2.2 \\
1.6 \\
4.2 \\
6.2 \\
2.4 \\
4.0 \\
\end{array}$ \\
\hline$B(7)$ & $\begin{array}{l}P_{p o s}(28.5) \\
P_{20}(27.5) \\
P_{20}(26.5)\end{array}$ & $\begin{array}{l}\text { yes } \\
\text { yes } \\
\text { yes }\end{array}$ & $\begin{array}{l}3.7 \\
3.5 \\
5.0\end{array}$ \\
\hline$A(5)$ & $P_{n}(10.5)$ & yes & 0.7 \\
\hline
\end{tabular}

Table 3. Candidate NO transitions for use in LIF measurements on HEG as determined from heated cell measurements at $1200 \mathrm{~K}$ and $1 \mathrm{kPa}$. ((yes) indicates a largely single line overlapping a minor component; intensity values are relative to the $R_{22}(27.5)$ value of 10 ) 


\subsection{Temperature measurements}

The relative intensities of several line pairs were measured in the heated cell filled with $500 \mathrm{~Pa} \mathrm{NO}$ at three temperatures $(1230,1000$ and $700 \mathrm{~K})$. Both laser beams were recorded simultaneously with the fluorescence images to apply corrections for fluctuations in intensity. Two single shot LIF images were corrected and combined to form a ratio $R$ of intensities (from which temperatures are calculated), and this process was repeated 100 times to give an average value and a scatter. Fig. 16 shows $\operatorname{In}(R)$ plotted against inverse temperature $1 / \pi_{\mathrm{TE}}$ as measured by a thermoelement, and a line whose slope corresponds to the energy separation of the two source NO energy levels, using published values from Hinz et $\mathrm{al}^{17}$. Since $T_{T E}$ varied by only $\pm 1 \mathrm{~K}$ during a measurement, the scatter seen in Fig. 16 cannot be due to a real temperature fluctuation, but must have another cause. There exists published data ${ }^{18}$ suggesting that shot-toshot fluctuations in the laser beam spectral intensity distribution occur, leading to difficulties in the combination of fluorescence images. (The effects of these fluctuations are, of course, reduced by averaging techniques, which cannot be used in HEG.) This topic is being addressed in further studies.

\subsection{TIME-RESOLVED MEASUREMENTS}

Since the energy transfer processes mentioned in 4.2 are time dependent, a deeper understanding of them requires both spectrally and time resolved measurements. The spectrally resolved fluorescence decay originating from the $R_{21}(17.5)$ and $R_{22}(27.5)$ lines was measured in the heated cell with NO density $\left(5 \times 10^{15} \mathrm{~cm}^{-3}\right)$ and temperature $(1170 \mathrm{~K})$, corresponding (to a first approximation) to HEG free stream conditions. A small camera gate (width $5 \mathrm{~ns}$ ) was moved with step $\Delta t-2 \mathrm{~ns}$ from pulse to pulse to later times after the laser pulse, leading to the rate of fluorescence decay, as averaged over very many laser pulses. (Problems due to pulse-to-pulse spectral fluctuations and a laser jitter and drift could not be allowed for here, so that some uncertainties remain regarding the accuracy of the measurement; it is planned to repeat these studies with faster detectors on just the one laser pulse, permitting "real time" capture of fluorescence decay with nanosecond resolution.) Nevertheless, the lifetime for the $D^{2} \Sigma^{+}\left(v^{\prime}=0\right)$ state was determined to be $19 \pm 2 \mathrm{~ns}$, which agrees quite well with the published value of Tsukiyama et $\mathrm{al}^{19}$.

\section{NO FLUORESCENCE QUENCHING}

NO fluorescence measured under HEG (or similar) conditions is expected to be partially quenched; this statement is supported by measurements carried out in the heated cell at HEG free stream densities and temperatures. For measurement of rotational temperatures using LIF two-line thermometry, the effects of quenching of the upper levels cancel out when forming signal ratios, but only if the quantum yield is independent of the rotational quantum number $K$, and only if one is sure that linear LIF (no saturation effects) can be assumed. Since $K$ lies approximately in the range 20 to 40 for the NO transitions accessed here, implying very large rotational energy level separations, the assumption that there is no $K$ dependence, which may well apply for small $K$, is no longer necessarily true here. Furthermore, to pursue the goal of also measuring NO concentrations in HEG, it is mandatory to look at the quenching behaviour of NO in the presence of different quench partners and at different temperatures. This will be tackled in three ways:-

1. LIF measurements on $\mathrm{NO}$ and $\mathrm{NO} / \mathrm{N}_{2}$ mixtures at different temperatures and pressures in the heated cell are being carried out, both time-integrated (using the available cameras) and time-resolved (using a new streak camera).

2. Since the flows in HEG are rich in atomic $O$ (see Table 1), and since it has been predicted that $O$ atoms are very efficient quenchers of NO A state fluorescence $e^{20}$, LIF quenching studies on the NO $D^{2} \Sigma^{+}\left(v^{\prime}=0\right)$ state in the presence of $O$ atoms are being undertaken in a small shock tube capable of delivering temperatures and densities comparable to the HEG free stream conditions. This work will be carried out using both photomultipliers and a streak camera, permitting time-resolved and time-/spectrally-resolved measurements, respectively. The first results on a shock tube study of $N O D^{2} \Sigma^{+}\left(v^{\prime}=0\right)$ state quenching led to values for $\sigma_{A r} \approx 40$ and $>16 \AA^{2}$ at 300 and $1980 \mathrm{~K}$, respectively, for quenching cross sections of NO by argon ${ }^{21}$. Callear et $a^{22}$ obtained a value of $\sigma_{N} \approx 25 \AA^{2}$.

3. Due to the complicated environment in HEG, and due to the uncertainties in the composition of the test gases, it is unlikely that LIF measurements from HEG can be easily corrected for quenching effects by using data obtained in the measurements obtained from 1. and 2. above. Using the techniques mentioned above for time-resolved LIF, in situ measurements in HEG will need to be carried out. 


\section{OTHER SPECTROSCOPIC TECHNIQUES}

Parallel to the work on LIF, where most of the effort has been placed, other non-imaging spectroscopic techniques are being implemented on HEG. Spectral analysis of the emission in the shocked region of a cylinder has been carried out to provide qualitative data on the source of the emission - it was shown to be $\mathrm{Fe}$ atoms - and to search for a spectral window for NO LIF measurements. An attempt to further analyse this data by comparing the measured spectra with simulated $\mathrm{Fe}$ spectra, calculated as a function of temperature, led only to approximate values for a gas temperature ${ }^{7}$. (Emission spectra are very difficult to analyse quantitatively; equilibrium amongst the various emitting electronic states is not always given, and line absorption or reversal due to cooler gases around the hot region is difficult to account for.)

However, the situation in the nozzle reservoir may be different. Here temperatures of over 9000 $\mathrm{K}$ and pressures of about $39 \mathrm{MPa}$ exist for $\mathrm{HEG}$ condition I (see Table l). Using quartz windows and optical fibres ${ }^{23}$, some of the radiation in this region has been brought outside to a photosensitive diode, where its intensity was measured as a function of time and compared with the pressure buildup (the at present only measurable property) in this region. It was also possible during a short test window to analyse this radiation spectrally over the wavelength range $200-500 \mathrm{~nm}$ using an optical multichannel analyser. Although the spectral resolution was not very good, a spectrum was obtained which showed no obvious strong discrete emission lines. In further work, the optical system will need an intensity calibration, and the spectral resolution will be improved, so that spectra can be measured and compared with the theoretical results for a blackbody radiator (if applicable here.)

First studies using the tunable laser diode absorption technique, as developed by Hanson and co-workers ${ }^{24}$, have been carried out ${ }^{25}$. A laser diode, with wavelength around $770 \mathrm{~nm}$, is tuned rapidly over the absorption profile of an $\mathrm{O}$ atom line originating from a transition between excited states $\left(3 s^{5} S \rightarrow 3 p^{5} P\right)$, so that a kinetic temperature (from the absorption bandwidth) and a flow velocity (from Doppler shift) over the lineof-sight in the HEG free stream can be determined. Since this absorption arises from electronically excited $\mathrm{O}$, its detection in the $\mathrm{HEG}$ free stream gases relies upon equilibrium not having been established amongst these highlevel electronic states. First attempts in the HEG could not detect an $\mathrm{O}$ atom absorption which was due to the free stream test gases.. The sensitivity of the technique is being improved, and, if necessary, other absorbing species will be examined.

\section{Z. CONCLUSIONS}

The study of gas properties which are in chemical (and perhaps vibrational) nonequilibrium using spectroscopic techniques is essential if the gas flows are to be properly understood. This further justifies the considerable effort that must be put into implementing these techniques on high enthalpy facilities where the conditions are extreme (high temperatures, nonequilibrium), where test times are short (single shot only is possible - this is a major disadvantage for quantitative work) and where the influence of extraneous luminosity can be disastrous (restricting the useful wavelengths for NO LIF, and necessitating the use of filters).

\section{REFERENCES}

1. W.H.Beck and K.Hannemann, An overview and appraisal of spectroscopic diagnostic techniques for HEG with the aid of sensitivity analyses of HEG model flow calculations (free stream and ELECTRE), DLR IB 222-93 C40, 1993.

2. D.Kastell, G.Eitelberg, M.Carl and S.Kortz, Phase step holographic interferometry in hypervelocity high temperature flows, DLR-IB 223-95 A 63 (1995).

3. R.M.Krek and G.Eitelberg, Classical characterisation of HEG (nozzle, freestream flow field), DLR-IB 223-94 A 50 (1994).

4. M.Scheer, Temperature and density measurements on hypersonic wind tunnels using laser induced fluorescence of NO, including a study of NO spectroscopy in the range 193-4 nm, using a novel heated cell, Diplom thesis, DLR, 1994.

5. M.Rosenhauer, Laserfluoreszenz Messungen an heissem NO im Göttinger Hochenthalpiekanal (HEG und in einer Kalibrierzelle, Diplom thesis, DLR, 1996.

6. W.H.Beck and M.Wollenhaupt, Search for spectral gaps in HEG, DLR-IB 223-95 A 44 (1995).

7. W.H.Beck, M.Müller and M.Wollenhaupt, Application of spectroscopic diagnostic techniques to studies on HEG: preparatory LIF work and emission spectroscopy results, 15th ICIASF, St.-Louis, France, IEEE Publ. 93CH3199-7 (1993). 
8. D.Kastell, M.Rosenhauer, M.Wollenhaupt, T.Müller, R.Krek, W.H.Beck and G.Eitelberg, Study of aerochemistry around a $70^{\circ}$ half angle blunted cone in $\mathrm{CO}_{2}$ environment in the high enthalpy shock tunnel. Part1: preparation of the tests, DLR-IB 223-95 C 17 (1995).

9. D.Kastell, R.Krek, M.Rosenhauer, M.Wollenhaupt, T.Müller, W.Beck and G.Eitelberg, ibid Part 2: Results, DLR-IB 22395 C 41 (1995).

10.M.Rosenhauer, M.Wollenhaupt, M.Scheer and W.H.Beck, LIF measurements in the wake of a blunt body in $\mathrm{HEG}$, in Second European Symposium on Aerothermodynamics for Space Vehicles, J.J.Hunt, ed., Noordwijk, Holland, P. 451 (1994).

11.D.Kastell, T.J.Horwath, and G.Eitelberg, Nonequilibrium flow expansion experiment around blunted a cone, ibid, p. 383 (1995).

12.M.Wollenhaupt, T.Müller and W.H.Beck, Cylinder experiments in the high enthalpy tunnel HEG. Part II: LIF studies, DLR-IB 22396 A 07 (1996).

13.H.Bito and W.H.Beck, High enthalpy heating test on KHI HOPE models using the high enthalpy shock tunnel (HEG) at the DLR Göttingen, DLR Contract Report (1995).

14.A.Hanamitsu, private communication of calculations carried out at Kawasaki Heavy Industries (1995).

15. (i)A.M.Wodtke, L.Huwel, H.Schluter, G.Meijer, P.Andresen and H.Voges, High sensitivity detection of NO in a flame using a tunable ArF laser, Opt. Letters 13, 910 (1988).

(ii)B.K.McMillin, M.P.Lee and R.K.Hanson, Planar laser-induced fluorescence imaging of nitric oxide in shock tube flows with vibrational nonequilibrium, Paper AIAA-90-1519 (1990).

(iii)D.C.Robie, J.D.Buck and W.K.Bischel, Bandwidth and tuning range of an ArF laser measured by $1+1$ resonantly enhanced multiohoton ionization of NO, Appl. Opt. 29, 3961 (1990).

16.M.Wollenhaupt and W.H.Beck, Hot cell tests, DLR-IB 223-95 A 62 (1995).

17.A.Hinz, J.S.Wells and A.G.Maki, Heterodyne frequency measurements on the nitric oxide fundamental band, J. Molec. Spect. 119: 120125 (1986).

18.B.K.McMillin, Instantaneous two-line PLIF temperature imaging of nitric oxide in supersonic mixing and combustion flowfields, HTGL Report T-287, Stanford University (1993).

19.K.Tsukiyama, T.Munakata, M.Tsukakoshi and T.Kasuya, Fluorescence lifetimes of NO $A^{2} \Sigma^{+}\left(v^{\prime}=3\right.$ and 4$), C^{2} \Pi\left(v^{\prime}=0\right)$ and $D^{2} \Sigma^{+}\left(v^{\prime}=0\right)$ studied by tunable VUV laser excitation, Chem. Phys. 121:55-62 (1988).
20.M.C.Drake and J.W.Ratcliffe, High temperature quenching cross sections for nitric oxide laser-induced fluorescence measurements, J.Chem.Phys. 98, 3850 (1993).

21.P.Andresen, W.H.Beck, G.Eitelberg, H.Hippler, T.J.Mclntyre, A.Riedl, T.Seelemann and J.Troe, A laser induced fluorescence system for the high enthalpy shock tunnel (HEG) in Göttingen, in Shock Waves, K.Takayama, ed., Proceedings 18th ISSW, Sendai Japan, 1991 p.657 (1992).

22.A.B.Callear, M.J.Pilling and I.W.M.Smith, Fluorescence of nitric oxide Part 5.-Mean lifetime in the $D^{2} \Sigma^{+}$state, Trans. Far. Soc. 64 , 2296 (1968).

23.O.Trinks, Optical studies in the HEG nozzle reservoir, DLR-IB 223-96, in preparation (1996).

24. L.C.Philippe and R.K.Hanson, Sensitive diode laser absorption technique for aerodynamic measurements, presented at 30th ASM, Reno, 1992, AIAA 92-0139 (1992).

25.W.Gillespie and W.H.Beck, Application of lineof-sight laser diagnostic techniques to the HEG shock tunnel, DLR-IB 223-94 A 52 (1994).

\section{ACKNOWLEDGEMENTS}

The financial support of ESA/CNES for much of the work here is gratefully acknowledged. The HERMES measurements were carried out under contract to Dassault Aviation (Velizy, France), and HOPE work was carried out for Kawasaki Heavy Industries (Japan) with the support of Mr H. Bito (NASDA, Japan). J.Lenz, W.Sader, D.Garbe and U.Frenzel provided valuable support on HEG. The laser apparatus was constructed by LaVision 2D Messtechnik GmbH (Göttingen, Germany) and the hot cell arose out of the Diplom-work from M.Scheer. 


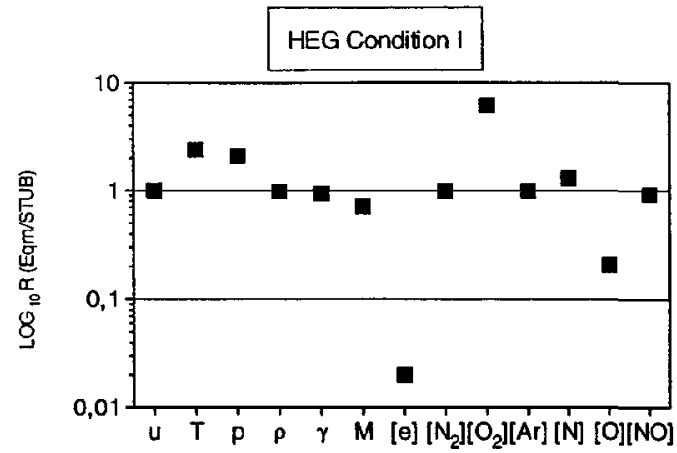

Fig. 1 Ratios of HEG free stream properties, calculated on the assumption of equilibrium relative to nonequilibrium (using STUB).

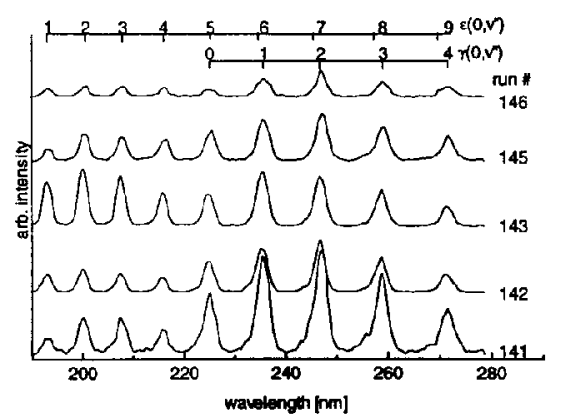

Fig. 3 Measured spectrally-resolved fluorescence signals after laser excitation of the $\varepsilon(0,1) R_{z}(27.5)$ transition for five runs at HEG conditions I (142), II $(141,145)$, III (143) and IV (146).

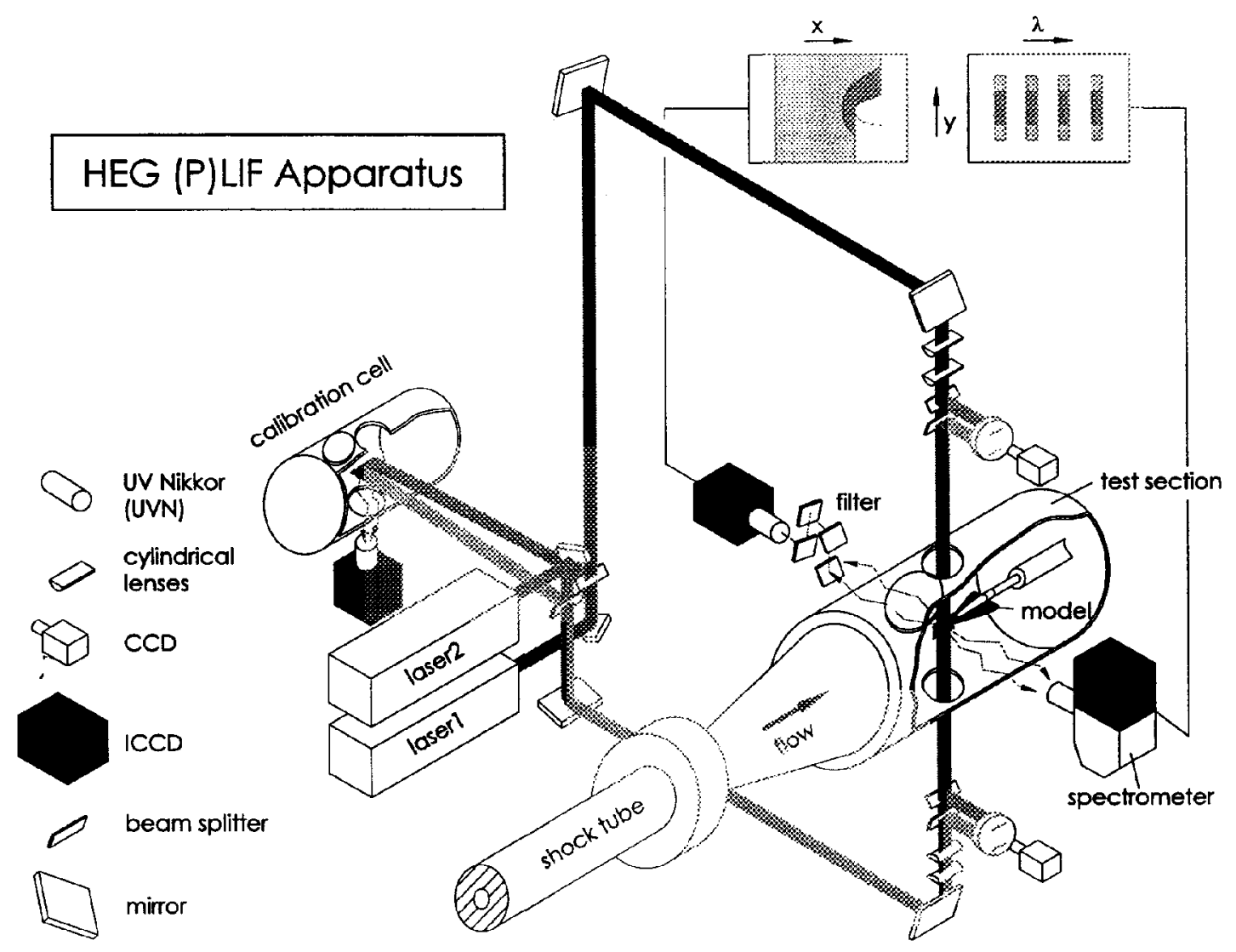

Fig. 2 Experimental setup for 2D (left camera systern) and spectrally-resolved (right camera system) NO LIF measurements on HEG. 
experimental setup

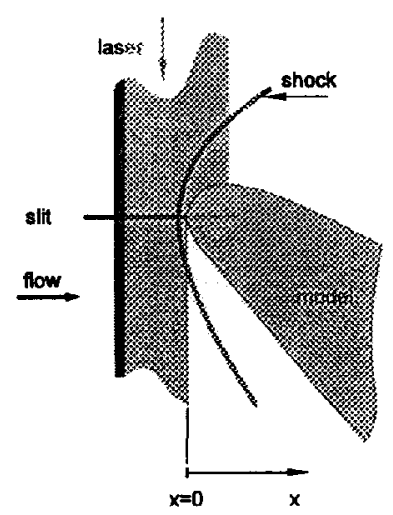

1-dimensionally and spectrally resolved laser induced fluorescence

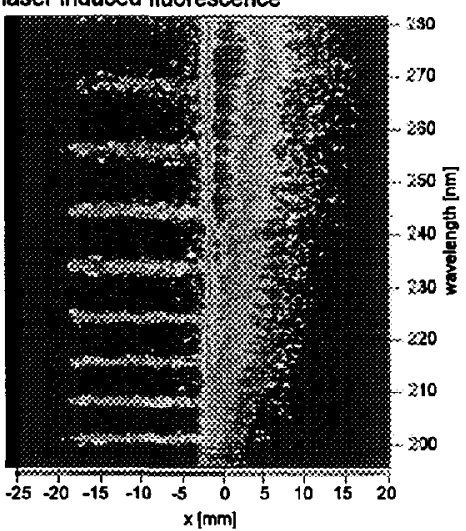

Spectra in thee free stream and behind the shock

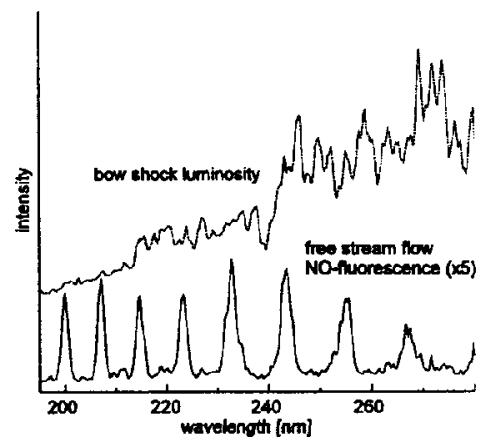

Arf laser excitation at $\tilde{v}=51639 \mathrm{~cm}^{1}$

Fig. 4 Spectrally-resolved NO LIF measurements in the stagnation region of a HERMES model at angle-of-attack $40^{\circ}$ in HEG at run condition I (21 MJ kg'; $41 \mathrm{Mpa}$ ). Left:- experimental side view, showing spectrometer slit position; middle:- NO LIF image, with fluorescence wavelength as ordinate and distance from HERMES bow ( $a t x=0$ ) as abscissa; right:- vertical profiles through the LIF image, taken in the freestream (lower) and shocked (upper) regions.

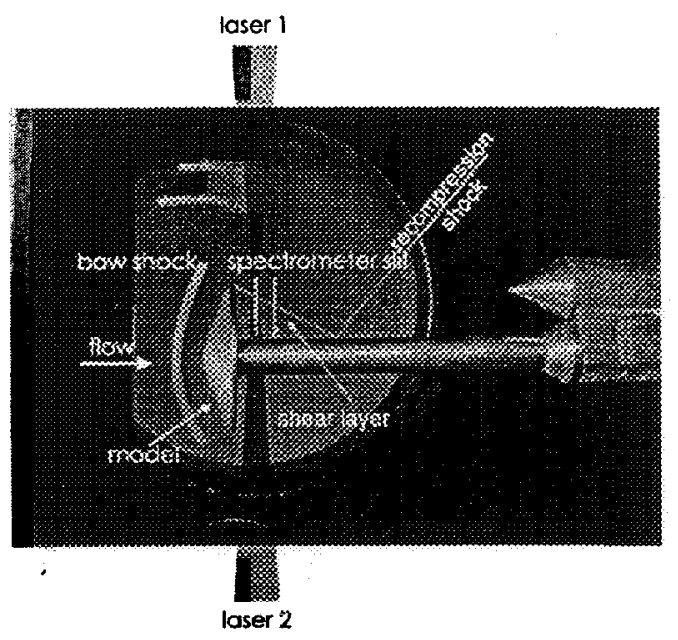

Fig. 5 Experimental setup for NO LIF measurements in the immediate wake of a blunted body in HEG, showing the two locations of the spectrometer slit relative to the position of the focussed laser beams.

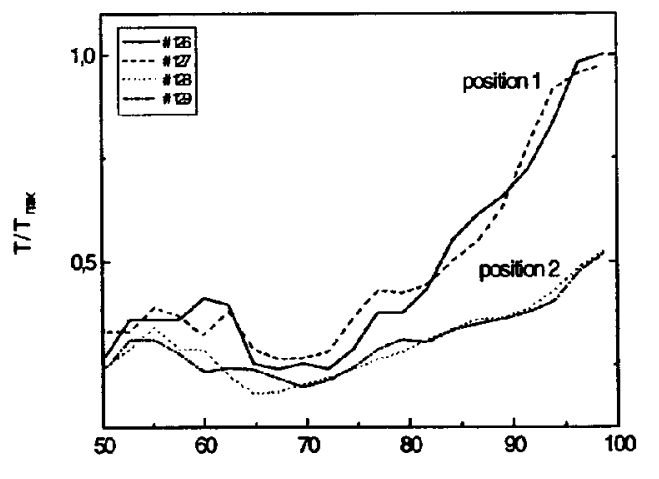

Fig. 6 Variation of NO rotational temperature $T$ in the wake of the blunted body, normalised relative to the maximum value $T_{\text {mained }}$ from $H E G$ run 126, at two downstream locations (see Fig. 5) for 4 HEG runs at condition III. 


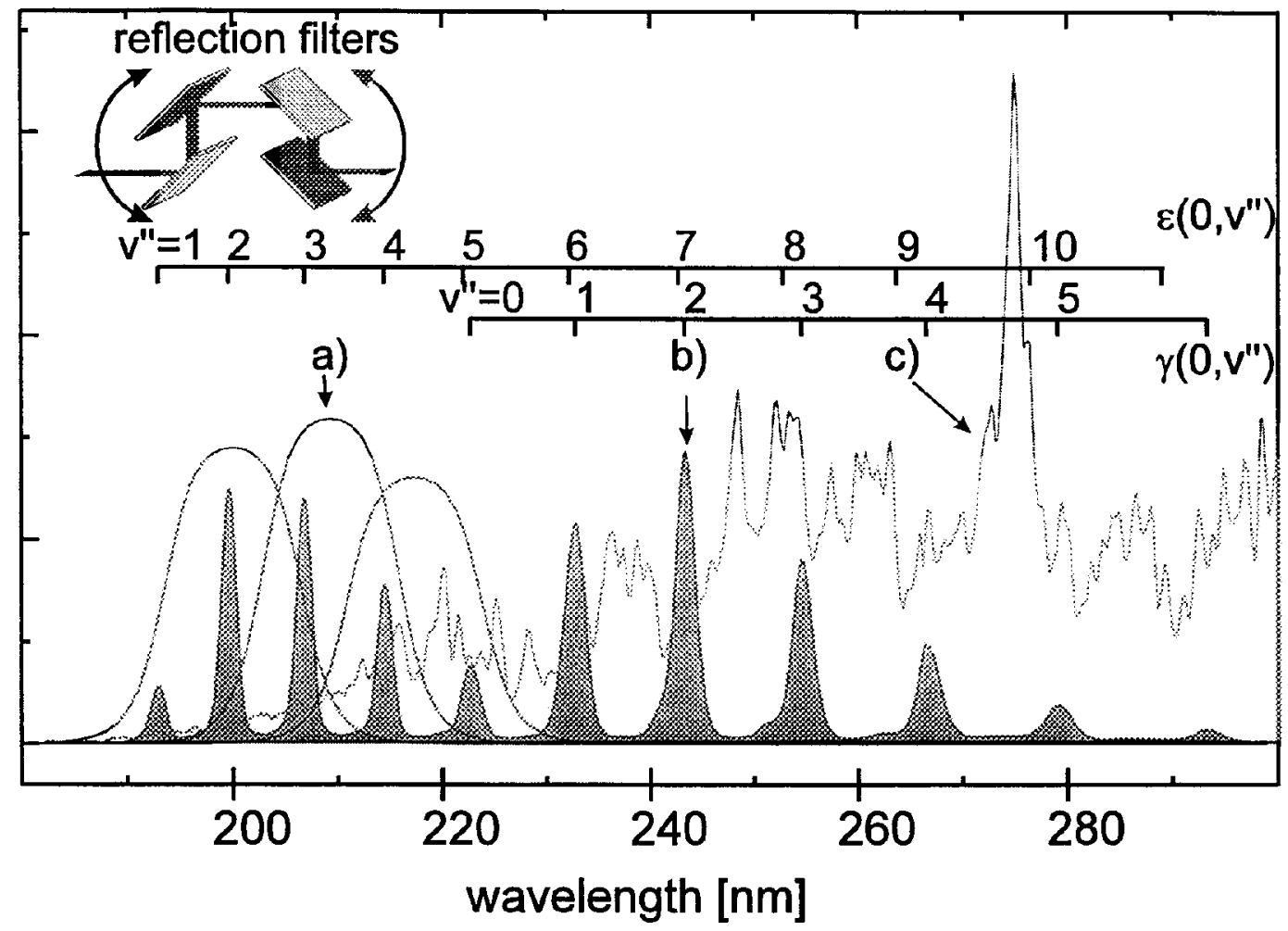

Fig. 7 Reflection filters for 2D NO PLIF measurements in HEG. (a) Variation of filter transmission curves for three different filter angles. (b) Typical NO fluorescence spectrum, as measured in the calibration cell (NO at $1200 \mathrm{~K}$ and $500 \mathrm{~Pa}$ ) after laser excitation of the $\varepsilon(0,1) R_{22}(27.5)$ transition. (c) Typical luminosity spectrum measured in HEG behind a cylinder bow shock ((b) and (c) are not to scale!).

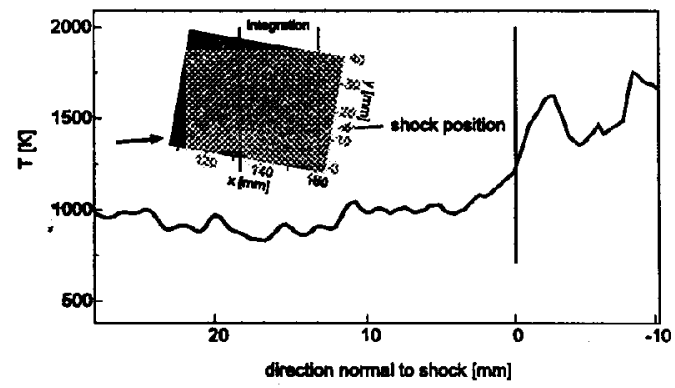

Fig. 8 NO LIF temperature profile across the recompression shock on the sting for HEG run 220 (condition III). A value of $1000 \mathrm{~K}$ (obtained from calculations) was assumed for the region above the shock.

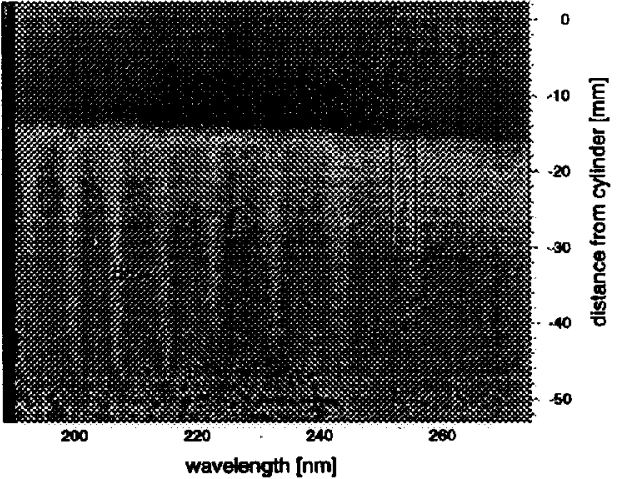

Fig. 9 Spectrally-resolved NO LIF measurement in the stagnation reglon of a cylinder model in HEG at run condition.I (run 236), showing saturated luminosity in regions 1 (cylinder) and 2 (shock-heated gases), scattered luminosity in region 3 (free stream flow) and NO

fluorescence in region 4 (free stream flow). The $45 \mathrm{~mm}$ laser sheet extends to the junction of regions 4 and 5 . Excitation is via the NO mixed line $\varepsilon(0,1)\left(Q_{11}(32.5)+\right.$ $Q_{21}(26.5)+R_{11}\left(26.5+R_{21}(21.5)\right)$ 


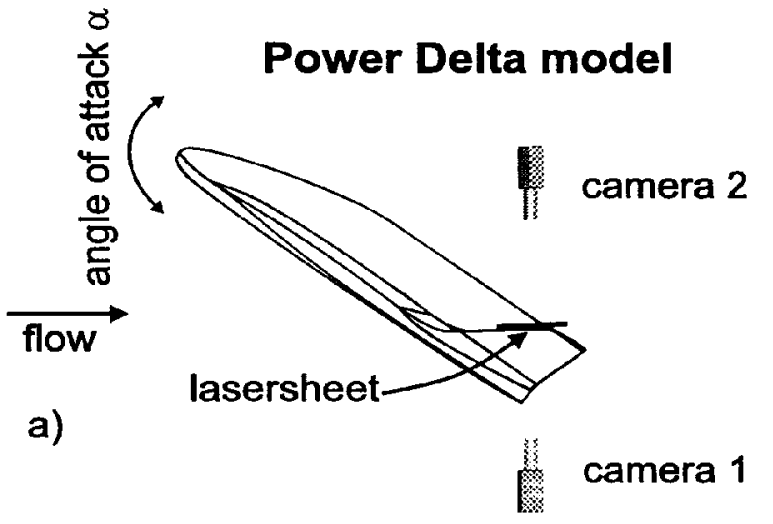

\section{Double Delta model}
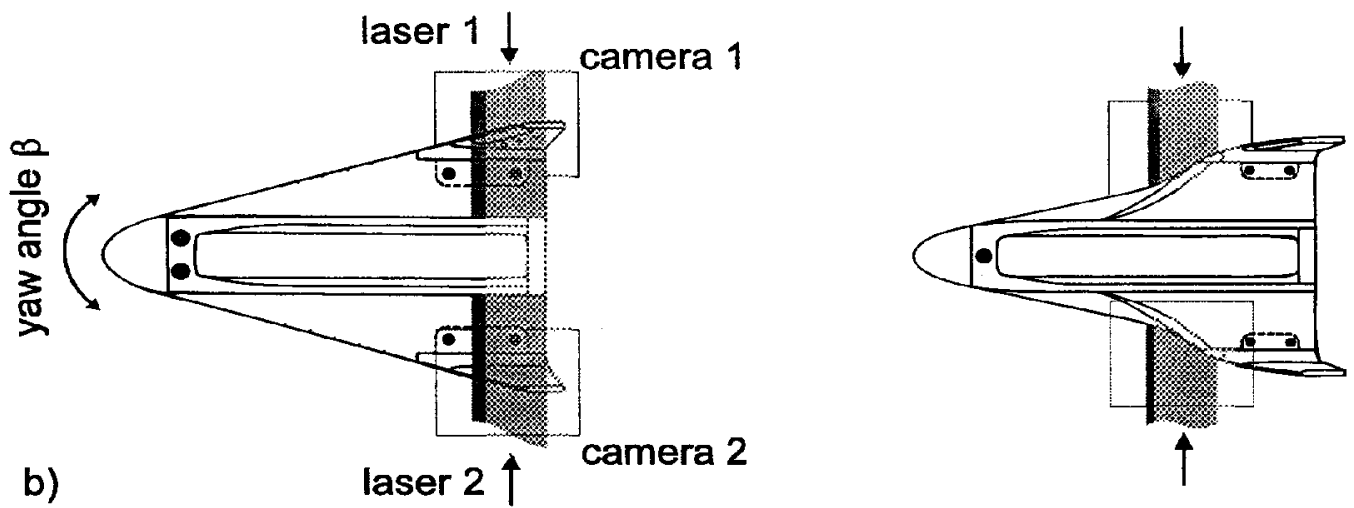

Fig. 10 Experimental setup for 2D NO PLIF measurements in HEG in the wing region of two candidate model shapes (power and double delta) for the Japanese space glider HOPE. The laser sheets were always positioned vertically in HEG. (a) Plan view, with model angle-of-attack $35^{\circ}$, and showing tilted laser sheet for the double delta model; (b) Side view (elevation), shown with yaw angle $0^{\circ}$.
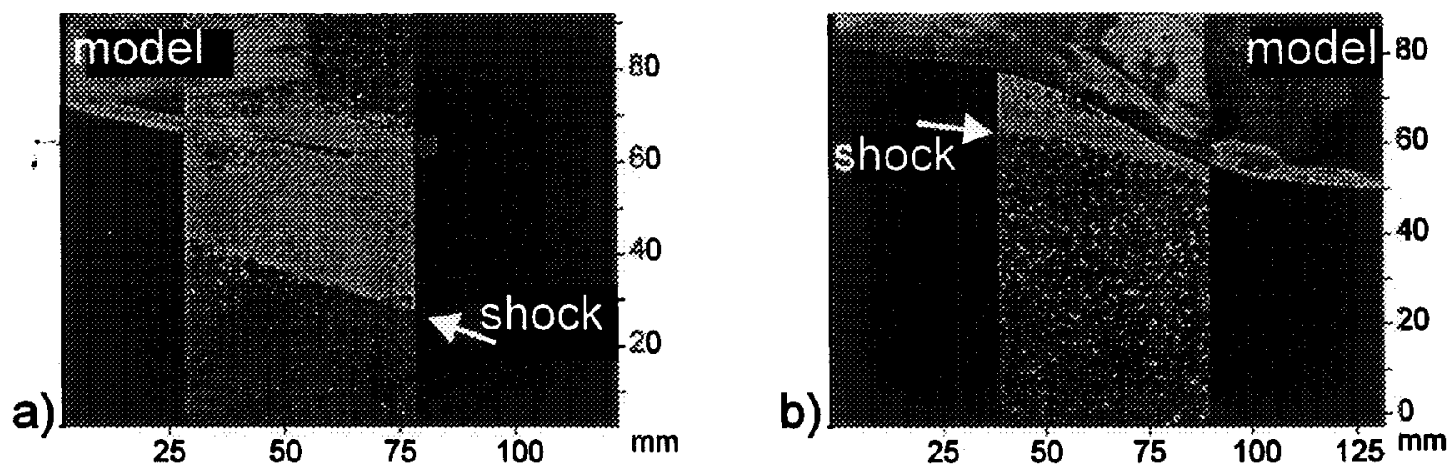

Fig. 11 2D NO PLIF images of the shock location in the vicinity of the wing of the power (a) and double (b) delta models with yaw angles 0 and $+3^{\circ}$, respectively, in HEG at condition $I$, after laser excitation of the $\varepsilon(0,1) R_{x}(27.5)$ transition. The fluorescence images have been overlapped with a model calibration picture to determine the shock location relative to the model. 
a)

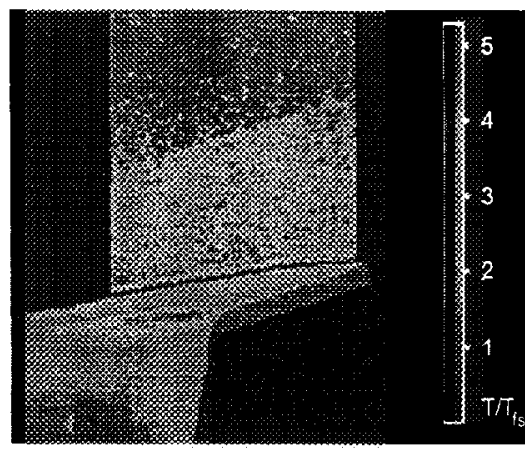

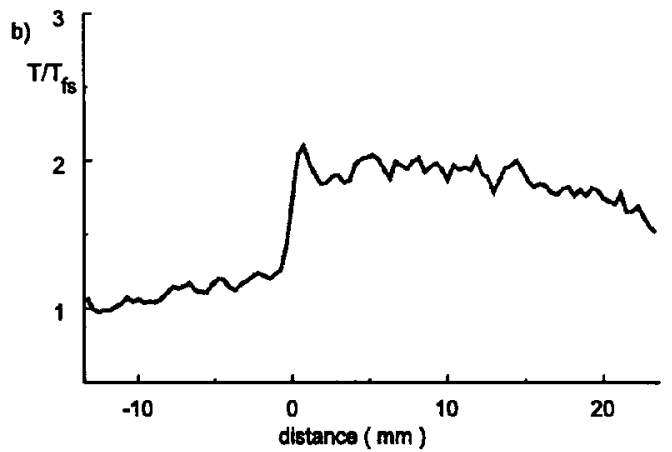

Fig. 12 NO rotational temperature distribution $T / T_{n}$, plotted relative to the free stream value $T_{n}$, in the vicinity of the power delta model wing tip fin for HEG run condition I. (a) $2 \mathrm{D}$ result overlapped with model shape; (b) Temperature profile $T \pi_{\mathrm{b}}$ across shock (distance $=0$ corresponds to shock location).

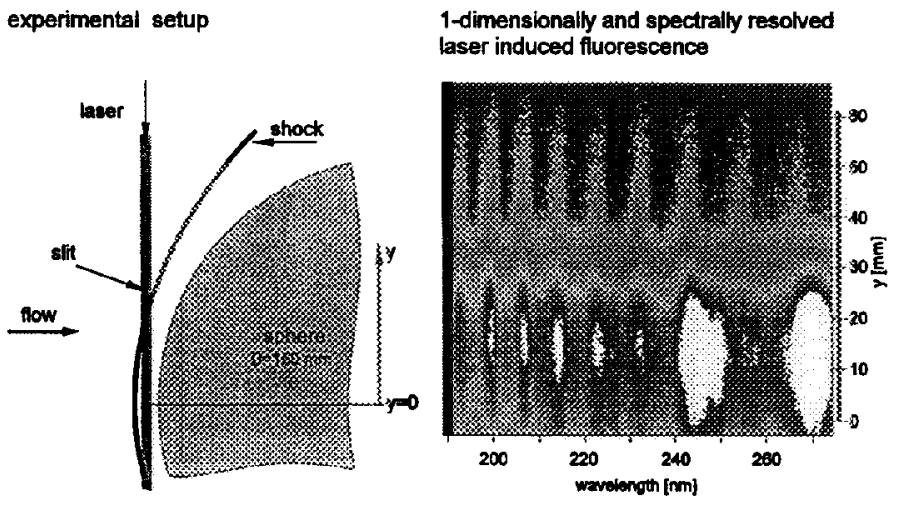

Spectra in the free stream and behind the shock

Fig. 13 Spectrally-resolved NO LIF measurements in the stagnation region of a spherical model in HEG at run condition I. Left:- experimental side view, showing different spectrometer slit position and laser beam orientation compared to HERMES measurements (Fig. 4); middle:- NO LIF image, with fluorescence wavelength as abscissa and distance y along slit ( $y=0$ corresponds to the stagnation line) as ordinate; right:- horizontal profiles through the LIF image, taken in the freestream (lower) and shocked (upper) regions.

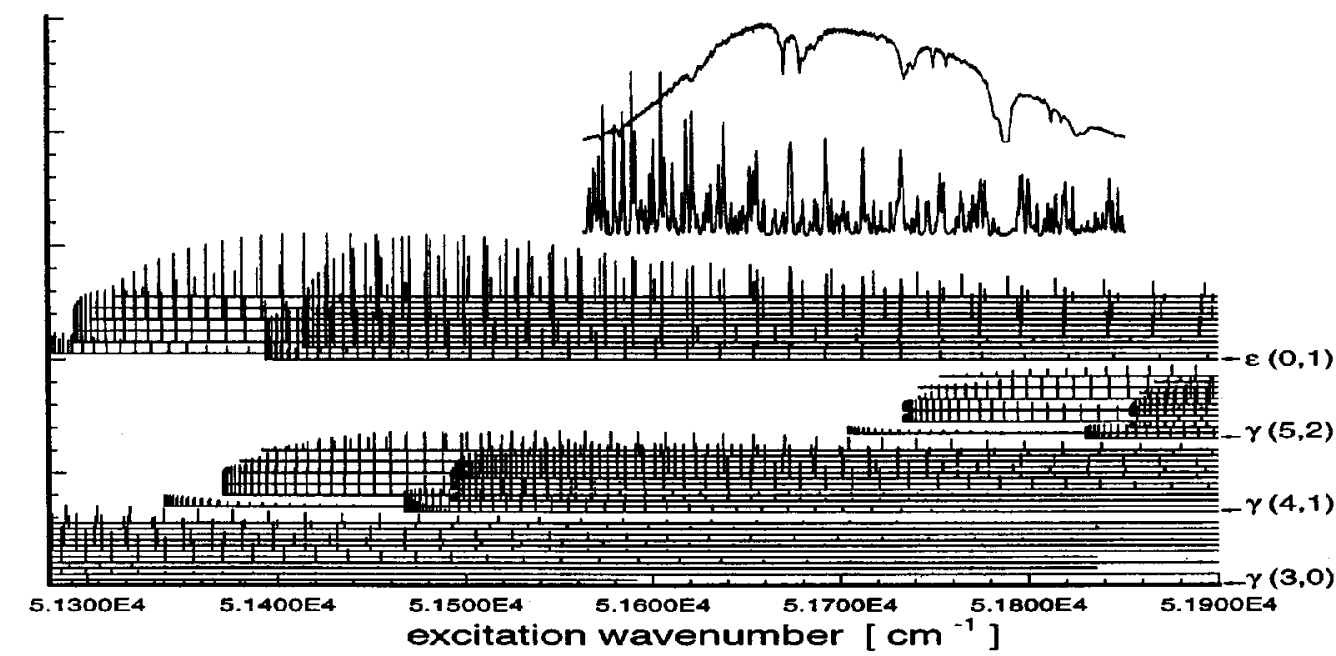

Fig. 14 Comparison between NO excitation spectrum (and laser locking curve) measured at $1200 \mathrm{~K}$ in the heated cell, top of figure, and theoretical spectra for the various $\gamma$ and $\varepsilon$ bands, including their branches, calculated over a wider spectral range. The profusion of lines from the various branches exemplifies how difficult it is in this wavelength range to find a single line not overlapped by others. 


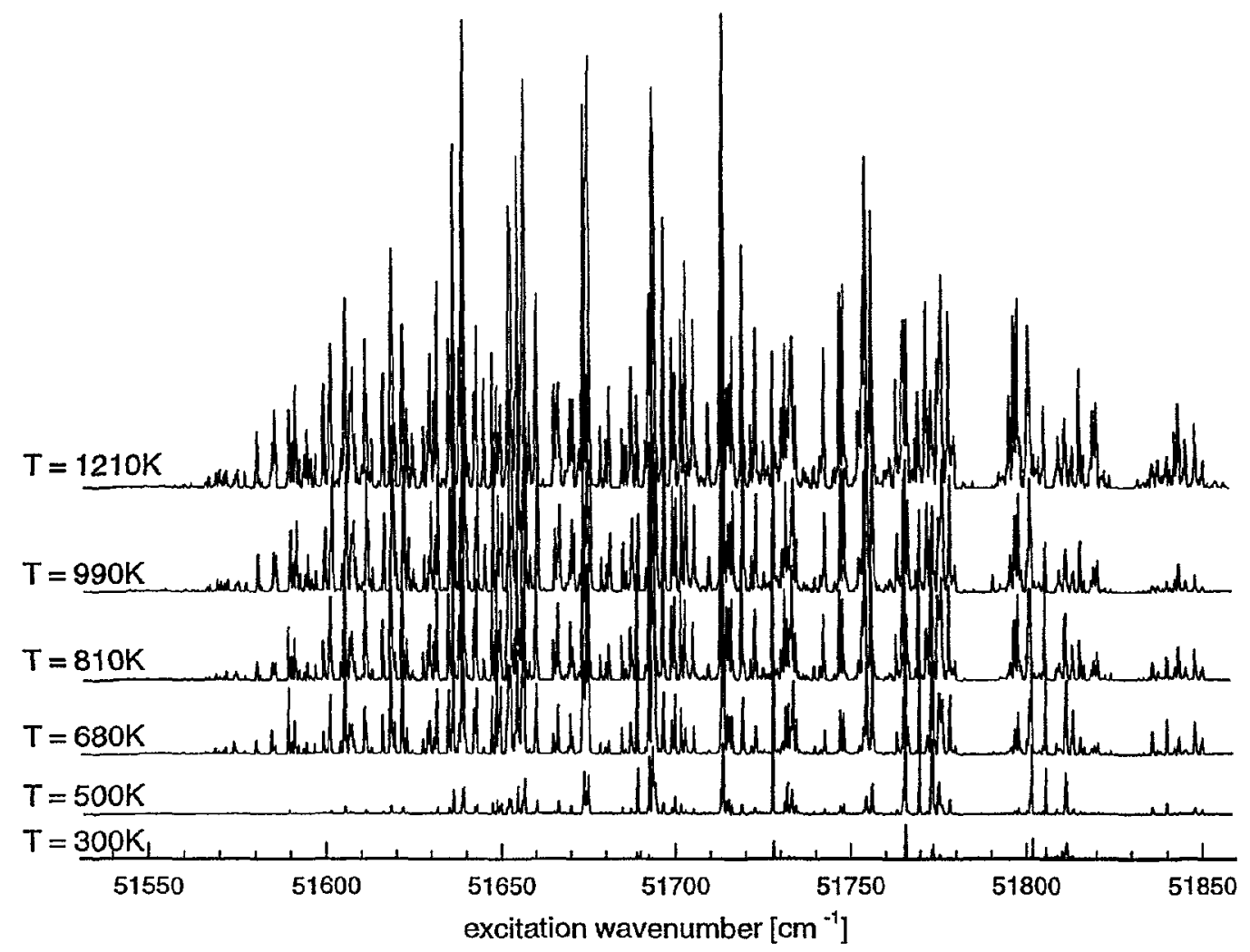

Fig. 15 NO excitation spectra, measured in the heated cell at various temperatures for a constant NO pressure of $1 \mathrm{kPa}$. Fluorescence signais are summed over the wavelength range $180-300 \mathrm{~nm}$. The increasing profusion of lines with increasing temperature can clearly be seen.
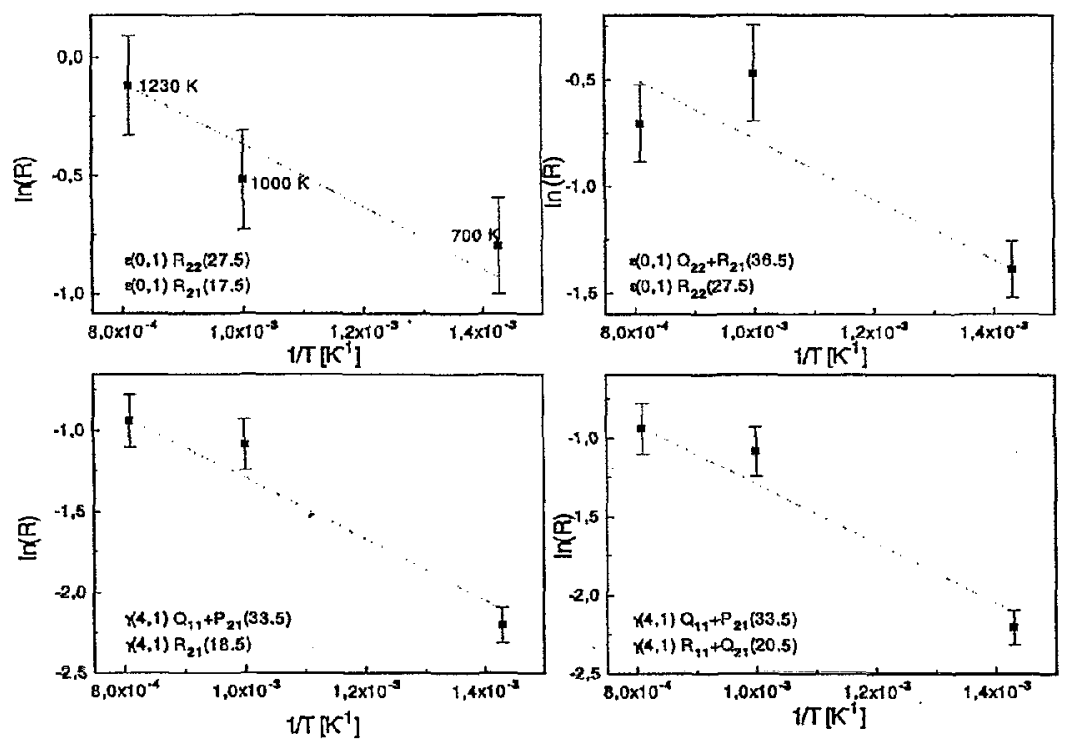

Fig. 16 Measured fluorescence ratio $\mathrm{R}$ (plotted as $\mathrm{hR}$ ) for four different pairs of NO transitions (shown in bottom left hand comer of each box), plotted as a function of inverse thermoelement temperature $(1 / T)$ in the heated cell. The plotted lines are Boltzmann plots with slopes derived from literature values (see text) for the energy separation between the two source energy levels. 
experimental setup

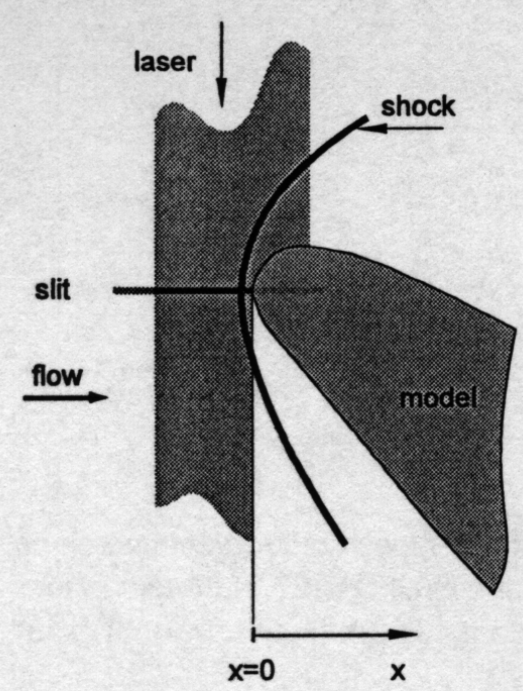

1-dimensionally and spectrally resolved laser induced fluorescence

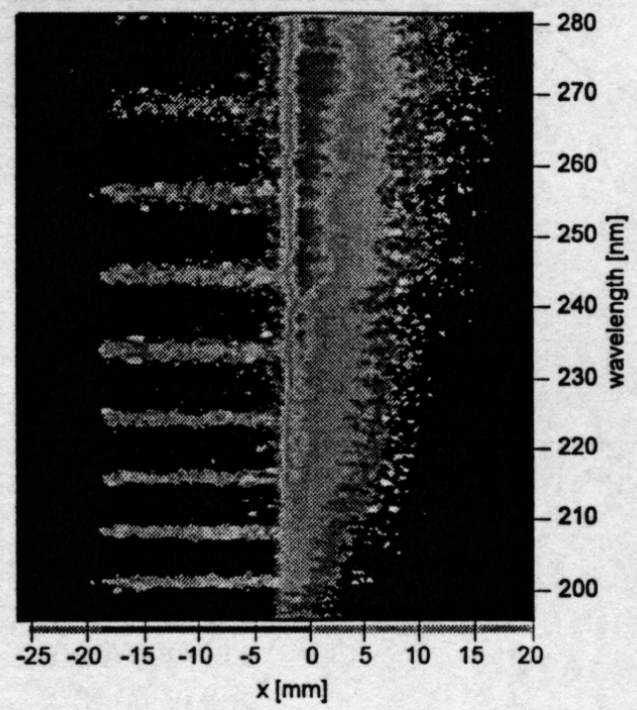

Spectra in thee free stream and behind the shock

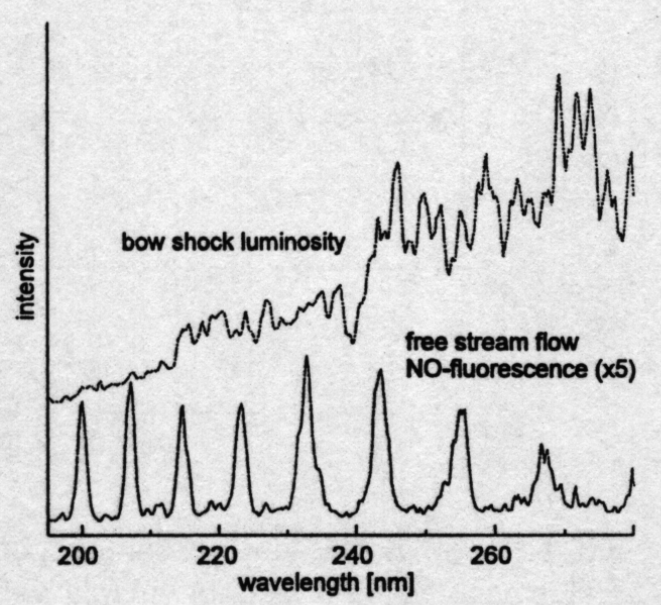

Arf laser excitation at $\tilde{v}=51639 \mathrm{~cm}^{-1}$

Fig. 4 Spectrally-resolved NO LIF measurements in the stagnation region of a HERMES model at angle-of-attack $40^{\circ}$ in HEG at run condition I (21 $\left.\mathrm{MJ} \mathrm{kg}^{-1} ; 41 \mathrm{Mpa}\right)$. Left:- experimental side view, showing spectrometer slit position; middle:- NO LIF image, with fluorescence wavelength as ordinate and distance from HERMES bow (at $x=0$ ) as abscissa; right:- vertical profiles through the LIF image, taken in the freestream (lower) and shocked (upper) regions. 


\section{laser 1}

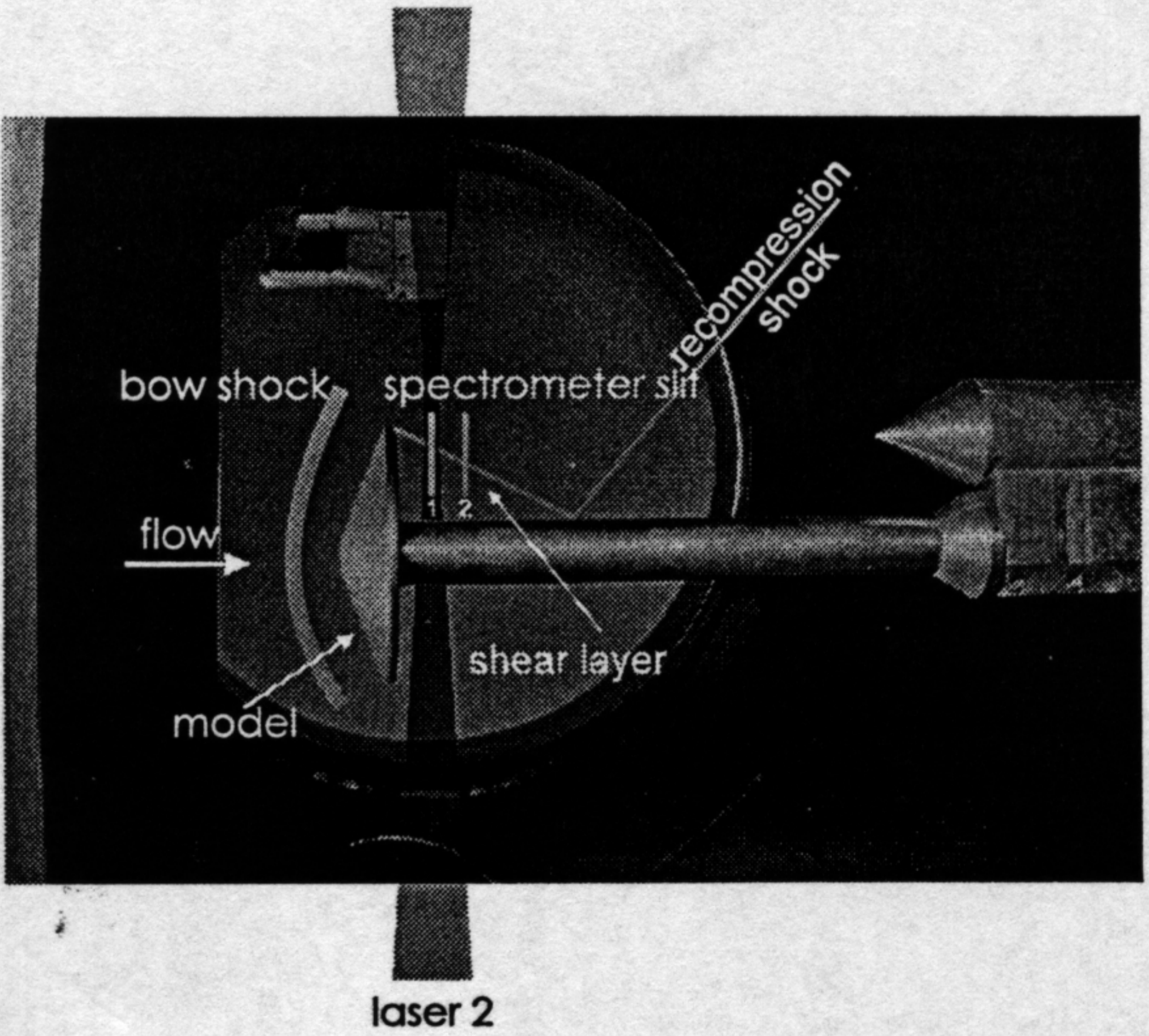

Fig. 5 Experimental setup for NO LIF measurements in the immediate wake of a blunted body in HEG, showing the two locations of the spectrometer slit relative to the position of the focussed laser beams. 


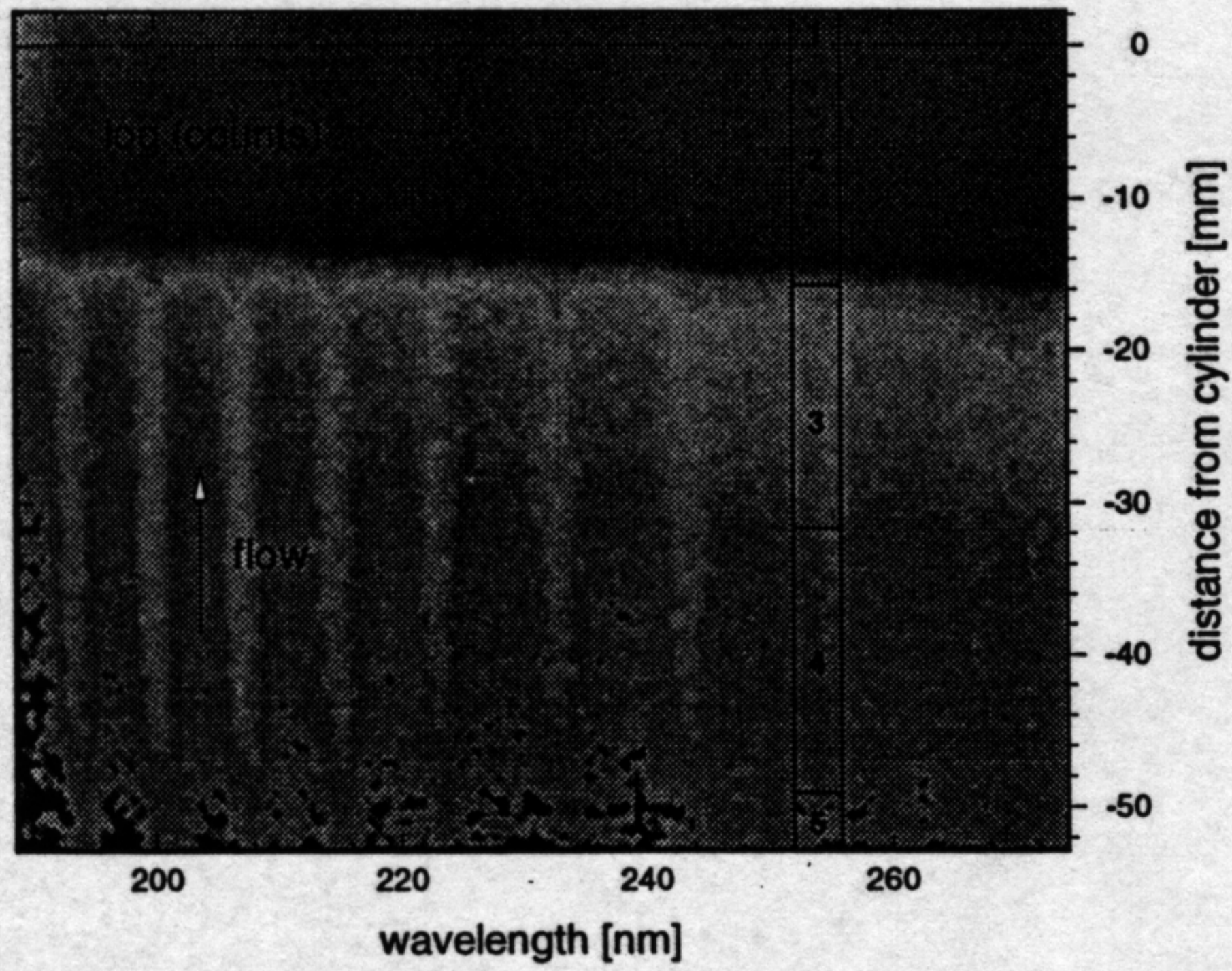

Fig. 9 Spectrally-resolved NO LIF measurement in the stagnation region of a cylinder model in HEG at run condition. I (run 236), showing saturated luminosity in regions 1 (cylinder) and 2 (shock-heated gases), scattered luminosity in region 3 (free stream flow) and NO fluorescence in region 4 (free stream flow). The $45 \mathrm{~mm}$ laser sheet extends to the junction of regions 4 and 5 . Excitation is via the NO mixed line $\varepsilon(0,1)\left(Q_{11}(32.5)+\right.$ $Q_{21}(26.5)+R_{11}\left(26.5+R_{21}(21.5)\right)$ 

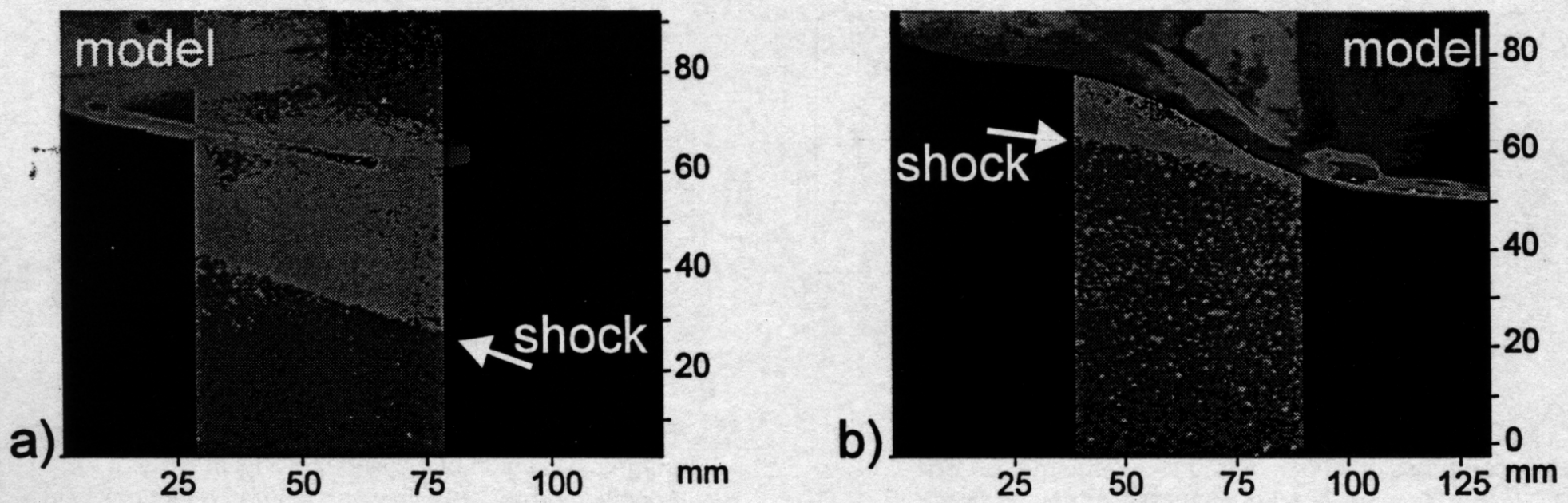

Fig. 11 2D NO PLIF images of the shock location in the vicinity of the wing of the power (a) and double (b) delta models with yaw angles 0 and $+3^{\circ}$, respectively, in $H E G$ at condition $I$, after laser excitation of the $\varepsilon(0,1) R_{22}(27.5)$ transition. The fluorescence images have been overlapped with a model calibration picture to determine the shock location relative to the model. 
a)

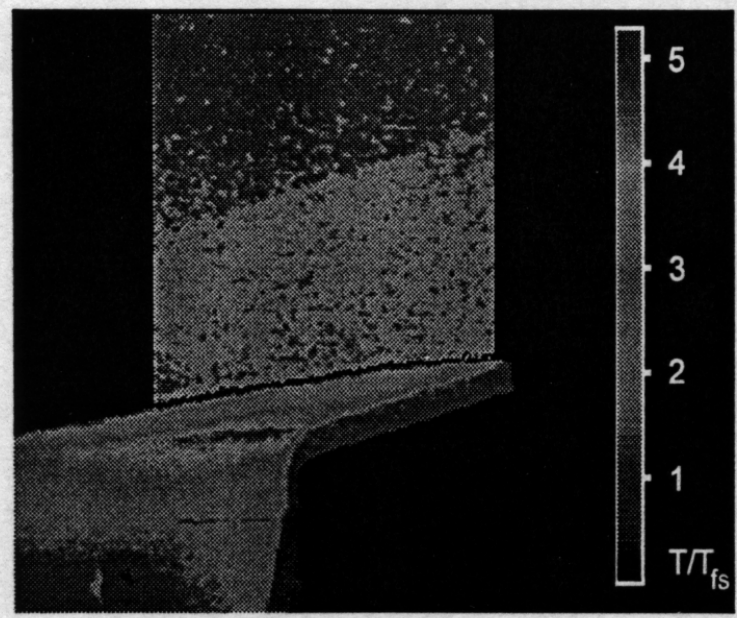

b)

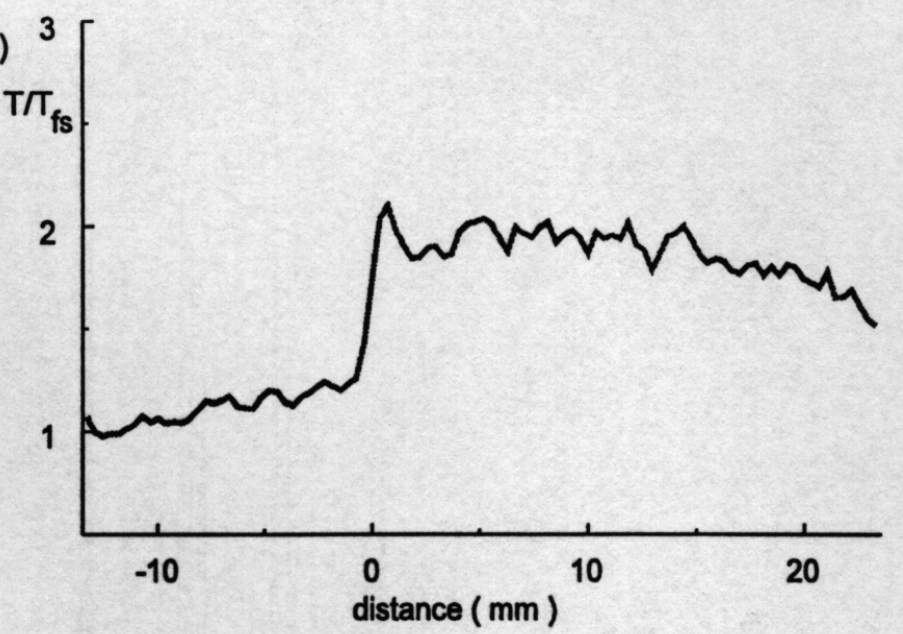

Fig. 12 NO rotational temperature distribution $T / T_{t s}$, plotted relative to the free stream value $T_{t s}$, in the vicinity of the power delta model wing tip fin for HEG run condition I. (a) $2 \mathrm{D}$ result overlapped with model shape; (b) Temperature profile $T / T_{\mathrm{ts}}$ across shock (distance $=0$ corresponds to shock location). 
experimental setup

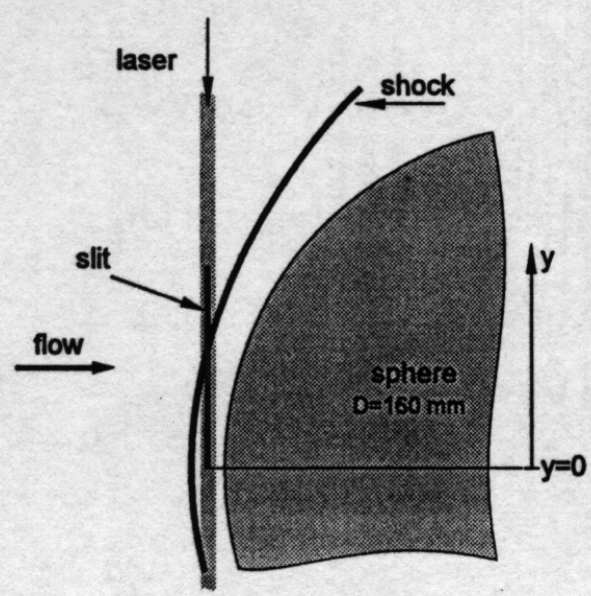

1-dimensionally and spectrally resolved laser induced fluorescence

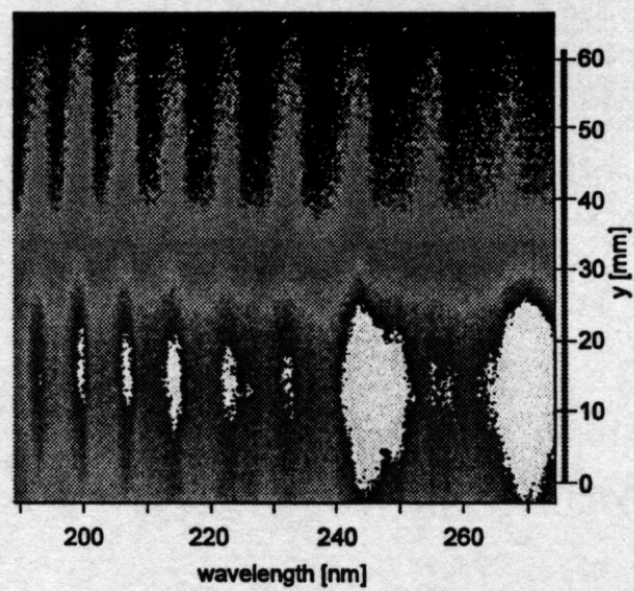

Spectra in the free stream and behind the shock

Fig. 13 Spectrally-resolved NO LIF measurements in the stagnation region of a spherical model in HEG at run condition I. Left:- experimental side view, showing different spectrometer slit position and laser beam orientation compared to HERMES measurements (Fig. 4); middle:- NO LIF image, with fluorescence wavelength as abscissa and distance $y$ along slit ( $y=0$ corresponds to the stagnation line) as ordinate; right:- horizontal profiles through the LIF image, taken in the freestream (lower) and shocked (upper) regions. 